\title{
Lepton-pair production in ultraperipheral collisions at AFTER@LHC
}

\author{
J.P. Lansberg, ${ }^{a}$ L. Szymanowski ${ }^{b}$ and J. Wagner ${ }^{b}$ \\ ${ }^{a}$ IPNO, Université Paris-Sud, CNRS/IN2P3, \\ F-91406, Orsay, France \\ ${ }^{b}$ National Centre for Nuclear Research (NCBJ), \\ Hoża 69, 00-681, Warsaw, Poland \\ E-mail: Jean-Philippe.Lansberg@in2p3.fr, Lech.Szymanowski@fuw.edu.pl, \\ Jakub. Wagner@ncbj.gov.pl
}

ABSTRACT: We investigate the potentialities offered by the study of lepton-pair production in ultraperipheral collisions at a fixed-target experiment using the proton and ion LHC beams. In these collisions, exclusive or semi-exclusive lepton-pair production can be used as luminosity monitor as well as a check of the equivalent-photon approximation, via the measurement of the Bethe-Heitler cross section. It can also serve as a probe of the inner hadron structure via the measurement of the lepton-pair azimuthal asymmetry which is sensitive to the timelike virtual Compton scattering. We also briefly discuss the possibility offered by the study of $\eta_{c}$ production. Finally, we outline the possibilities for lepton-pair production by Odderon-Pomeron fusion in exclusive $p p$ collisions.

Keywords: Hadronic Colliders, Heavy Ion Phenomenology

ArXiv EPrint: 1504.02733 


\section{Contents}

1 Introduction 1

2 Ultraperipheral collisions in a high-energy fixed-target experiment $\quad 2$

2.1 Generalities on photon-induced reactions in ultraperipheral collisions 2

2.2 Photon fluxes 3

2.3 Fluxes and the rapidity dependence of the produced particles 6

$\begin{array}{ll}2.3 .1 & \text { Single-photon case }\end{array}$

2.3.2 Double-photon case 8

3 Lepton-pair production: energy, invariant-mass, rapidity and transverse$\begin{array}{lr}\text { momentum dependencies } & 9\end{array}$

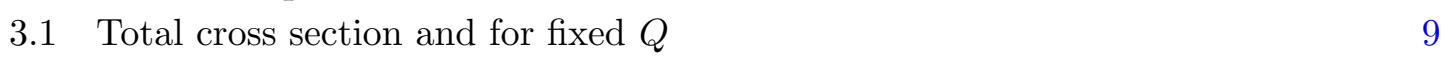

$\begin{array}{lll}3.2 & \text { Production at nonzero transverse momenta } & 12\end{array}$

4 Timelike Compton scattering or exclusive photoproduction of a dilepton 13

5 Exclusive lepton-pair hadroproduction via odderon-pomeron fusion $\quad 17$

$\begin{array}{llr}6 & \text { Conclusion } & 20\end{array}$

$\begin{array}{ll}\text { A Kinematics } & 21\end{array}$

B Compton form factors and generalised parton distributions 22

\section{Introduction}

With the advent of RHIC and the LHC, the first experimental studies of Ultra-Peripheral Collisions (UPC) have successfully been carried out. The STAR collaboration first measured the $\rho_{0}$ production cross section in AuAu UPC [1], then they measured the di-electron production cross section in UPC [2]. A little later the PHENIX collaboration released their first study of the $J / \psi$ cross section as well as high-mass di-electron production [3]. More recently, further studies have been carried out at the LHC by the ALICE collaboration [4, 5].

Attempts to isolate UPC in InIn collisions in the fixed-target mode at the SPS have been made. ${ }^{1}$ They were not conclusive, most probably because of the limited nucleonnucleon centre-of-mass (cms) energy, on the order of $20 \mathrm{GeV}$, resulting in typical photonnucleon cms energies below $3 \mathrm{GeV}$.

In this context, we investigate the possibility to study lepton-pair production in ultraperipheral collisions at a fixed-target experiment using the proton and ion LHC beams [6]

\footnotetext{
${ }^{1}$ P. Ramalhete, PhD. thesis, April 2009.
} 
- referred thereafter to as AFTER@LHC. In such collisions, one can investigate specific reactions where one of the colliding particle serves as a (coherent) emitter of a photon and the other serves as a target. Such photon-hadron collisions can be (semi-)exclusive, resulting, for instance, in the sole production of a lepton pair. This process can be used to monitor the experiment luminosity since it mainly comes from the Bethe-Heitler (BH) process [7], whose cross section is well-known. By looking at the target rapidity region, it can also serve to scan the domain of validity of the Equivalent-Photon Approximation (EPA). Moreover it can also help to probe the inner structure of the target, through the interference between the BH process and the Timelike Compton Scattering (TCS) [8-12]. Such an interference - measurable via the analysis of the azimuthal anisotropy — indeed involves contributions from the Generalised Parton Distributions (GPD) [13-20].

The structure of this article is as follows. In section 2, we present the main characteristics of the UPCs and the corresponding photon fluxes in a fixed-target mode on the LHC beams. In section 3, we briefly discuss the cross sections for production of lepton pair via the $\mathrm{BH}$ process. In section 4 , we discuss how the contribution from TCS can be extracted and how they can help to unravel information about the inner proton structure. In section 5, we briefly discuss the potential competing hadronic process resulting from photon-odderon fusion. Finally, we present our outlooks and conclusions.

\section{Ultraperipheral collisions in a high-energy fixed-target experiment}

\subsection{Generalities on photon-induced reactions in ultraperipheral collisions}

Relativistically moving charged hadrons are accompanied by electromagnetic fields which can effectively be used as quasi-real-photon beams. At very high energies, these photons are energetic enough to initiate hard reactions, just as in lepton-proton colliders.

The virtuality, $q^{2}=-Q^{2}$, of these photons is small, $Q^{2} \lesssim 1 / R^{2}$, where $R$ is the radius of the charge. More precisely, $Q \lesssim 0.28 \mathrm{GeV}$ for protons $(R \approx 0.7 \mathrm{fm})$ and $Q \lesssim 0.06 \mathrm{GeV}$ for nuclei $\left(R_{A} \approx 1.2 A^{1 / 3} \mathrm{fm}\right)$ with a mass number $A>16$. These photons, which are emitted coherently, are almost on mass shell, and their emission can be theoretically treated in the EPA (see e.g. [21]).

Seen from a target at rest, the energy of these photons can become significant if the energy of the moving charge, i.e. the beam energy, becomes ultrarelativistic, as at the LHC. At rest, the coherent photon cloud of an heavy ion is on the order of $30 \mathrm{MeV}$. Boosted a few thousand times $\left(\gamma_{\mathrm{Pb}} \simeq 2940\right)$, these photons have an energy close to $100 \mathrm{GeV}$ in the laboratory frame. It is of course much less than what can be achieved at the LHC in the collider mode, but close to the experimental condition at RHIC with colliding beams of $100 \mathrm{GeV}$. It is anyhow enough to produce hard dileptons as well as vector mesons.

The energy spectrum of these photons depends on the boost with respect to the observer as well as the impact parameter $b$ - it is understood that the observer or the probe is outside the charge distribution. Using the EPA method, one gets [22] that the flux as function of the photon momentum $k$, of $b$ and $\gamma$ (the Lorentz factor of the hadron - or 
nucleus - in the frame where $k$ is measured) reads

$$
\frac{d n}{d k d^{2} b}=\frac{Z^{2} \alpha_{\mathrm{em}} \omega(b, k)^{2}}{\pi^{2} k b^{2}}\left[K_{1}^{2}(\omega(b, k))+\frac{1}{\gamma^{2}} K_{0}^{2}(\omega(b, k))\right],
$$

where $\alpha_{\mathrm{em}}$ is the QED coupling, $Z$ is the nucleus charge, $\omega(b, k)=k b / \gamma$ and $K_{1,2}$ are modified Bessel functions of the second kind.

One usually assume that $b$ cannot be smaller than $R$. Otherwise, (i) one cannot consider the entire nucleus charge $Z$, (ii) hadronic interactions may be more important that the photon-induced ones, (iii) the probability for the colliding objects to break-up may also be important. For $b \gtrsim R$, the strong suppression of the flux by the Bessel functions when $\omega$ gets of the order of unity implies that $k$ should be smaller than inverse radius times the Lorentz factor $\gamma$. The larger $\gamma$ is and the smaller the emitter is, the harder the flux is. We also note that the energy spectrum $k d n / d k$ is constant for $k / \gamma$ fixed. To fix the idea, one usually considers a maximum photon momentum $k^{\max } \simeq \frac{\hbar c}{R_{\text {emitter }}}$ below which the emission are likely coherent and therefore characterised by a flux proportional to $Z^{2}$. This quantity should not thus be considered as a sharp cut-off above which photon emissions are forbidden. If the photon is considered in the hadron cms, one has $\gamma=\sqrt{s_{N N}} /\left(2 m_{N}\right)(\equiv$ $\left.\gamma^{\mathrm{cms}}\right)$. If the photon is considered in the target $B$ rest frame, $\gamma=s_{N N} /\left(2 m_{N}^{2}\right)\left(\equiv \gamma^{A \rightarrow B}\right)$. Table 1 summarises the relevant parameters characterising ultra-peripheral collisions at AFTER@LHC, at RHIC and a SPS in fixed-target mode.

For comparisons with photon-induced reactions in the more conventional lepton-hadron collisions, it is usually more instructive to look at the maximum of the $\gamma N \mathrm{cms}$ energy, $\sqrt{s_{\gamma N}^{\max }}$. To do so, we "boost" $k^{\max }$ in the "target" nucleon rest frame, to obtain $E_{\gamma \text { max }}^{\mathrm{N} \text { rest }}=\gamma^{A \rightarrow B} k^{\max }$, where $\sqrt{s_{\gamma p}}=\sqrt{2 E_{\mathrm{N} \text { rest }}^{\max } m_{N}}=\sqrt{s_{N N} k^{\max } / m_{N}}$. We note that the photon-energy "cut-off" obtained with these dimensional arguments for $\mathrm{Pb} p$, i.e. $74 \mathrm{GeV}$, is remarkably close to the peak in the energy spectrum obtained using a more realistic model in a recent study of the Bremsstrahlung spectrum of ions in AFTER@LHC [26], i.e. $80 \mathrm{GeV}$.

\subsection{Photon fluxes}

Taking into account the smallest possible impact parameter for a given colliding system, $p p, p A$ or $A B$, as well as the charge distribution through a form factor in the proton case, one obtains different formulae for the flux integrated in $b$. In fact, the photon fluxes do not formally factorise since $b_{\text {min }}$ depends on the radius of both colliding objects, except in $e p$ collisions, where one can reasonably neglect the electron radius.

Along these lines, one should normally have for $p p$ collisions, $b_{\min } \simeq 2 \times R_{p}$; for $p A$ collisions, $b_{\text {min }} \simeq R_{p}+R_{A}$; and for $A B$ collisions $b_{\text {min }} \simeq R_{A}+R_{B}$. This is what we have chosen in table 1 . Whereas it is acceptable to approximate $R_{p}+R_{A}$ to $R_{A}$, it does not seem justifiable to use $R_{\mathrm{Pb}}$ for $\mathrm{PbPb}$ collisions, for instance. In addition, in $p A$ collisions, it is also problematic to use a different $b_{\min }$ when one considers the proton emission or the ion emission. In both cases, one should use $R_{p}+R_{A}$, or perhaps $R_{A}$.

\footnotetext{
${ }^{2}$ In fact, the procedure looks more as if the emitter is Lorentz contracted as $1 / \gamma$, rather than the photon momentum boosted. The results are however similar once one considers the emitted photon as slightly off-shell, with a momentum, in the rest frame of the emitter, as $\left(k^{\max }, 0,0,0\right)$.
} 


\begin{tabular}{|c|c|c|c|c|c|c|c|c|c|c|c|c|}
\hline \multicolumn{2}{|c|}{$\begin{array}{r}\text { System target } \\
\text { thick- } \\
\text { ness } \\
(\mathrm{cm}) \\
\end{array}$} & $(\mathrm{GeV})$ & $\left(\mathrm{pb}^{-1} \mathrm{yr}^{-1}\right)$ & $(\mathrm{GeV})$ & $(\mathrm{GeV})$ & $\left(\frac{\sqrt{S} N N}{2 m_{N}}\right)$ & $\left(\frac{s_{N N}}{2 m_{N}^{2}}\right)$ & $(\mathrm{MeV})$ & $E_{\gamma}^{\mathrm{A} / \mathrm{Br} \mathrm{rax}}$ & $\sqrt[\operatorname{est}]{s_{\gamma_{N}}^{\max }}$ & $\begin{array}{l}E_{\gamma \text { max }}^{\mathrm{cms}} \\
(\mathrm{GeV})\end{array}$ & $\begin{array}{l}\sqrt{s_{\gamma \gamma}^{\max }} \\
(\mathrm{GeV})\end{array}$ \\
\hline \multicolumn{13}{|c|}{ AFTER@LHC } \\
\hline$p p$ & 100 & 115 & $2.0 \times 10^{4}$ & 7000 & $m_{N}$ & 61.0 & 7450 & 141 & 1050 & 44 & 8.6 & 17 \\
\hline$p \mathrm{~Pb}$ & 1 & 115 & 160 & 7000 & $m_{N}$ & 61.0 & 7450 & 25.3 & 188 & 19 & 1.5 & 3.1 \\
\hline$p d$ & 100 & 115 & $2.4 \times 10^{4}$ & 7000 & $m_{N}$ & 61.0 & 7450 & 69.5 & 517 & 31 & 4.2 & 8.5 \\
\hline $\mathrm{PbPb}$ & 1 & 72 & 7. $\times 10^{-3}$ & 2760 & $m_{N}$ & 38.3 & 2940 & 13.9 & 40.7 & 8.8 & 0.53 & 1.1 \\
\hline $\mathrm{Pb} p$ & 100 & 72 & 1.1 & 2760 & $m_{N}$ & 38.3 & 2940 & 25.3 & 74.2 & 12 & 0.97 & 1.9 \\
\hline $\operatorname{Ar} p$ & 100 & 77 & 1.1 & 3150 & $m_{N}$ & 40.9 & 3350 & 41.1 & 138 & 16 & 1.7 & 3.4 \\
\hline $\mathrm{O} p$ & 100 & 81 & 1.1 & 3500 & $m_{N}$ & 43.1 & 3720 & 53.0 & 197 & 19 & 2.3 & 4.6 \\
\hline \multicolumn{13}{|l|}{ RHIC } \\
\hline$p p$ & $\mathrm{n} / \mathrm{ap}$ & 200 & 12 & 100 & 100 & 106 & 22600 & 141 & 3190 & 77 & 15 & 30 \\
\hline $\mathrm{AuAu}$ & $\mathrm{n} / \mathrm{ap}$ & 200 & $2.8 \times 10^{-3}$ & 100 & 100 & 106 & 22600 & 14.2 & 320 & 25 & 1.5 & 3.0 \\
\hline \multicolumn{13}{|l|}{ SPS } \\
\hline InIn & $\mathrm{n} / \mathrm{av}$ & 17 & $\mathrm{n} / \mathrm{av}$ & 160 & $m_{N}$ & 9.23 & 170 & 16.9 & 2.87 & 2.4 & 0.16 & 0.31 \\
\hline $\mathrm{PbPb}$ & $\mathrm{n} / \mathrm{av}$ & 17 & $\mathrm{n} / \mathrm{av}$ & 160 & $m_{N}$ & 9.23 & 170 & 13.9 & 2.36 & 2.1 & 0.13 & 0.26 \\
\hline
\end{tabular}

Table 1. Relevant parameters for $A B$ UPCs at AFTER@LHC, at RHIC and at SPS: (i) nucleonnucleon cms, $\sqrt{s_{N N}}$ (ii) luminosity, $\mathcal{L}_{A B}$, (iii-iv) colliding hadron energies, $E_{A, B}^{\text {lab }}$, in the laboratory frame, (v) Lorentz factor between the colliding-hadron rest frame and cms, $\gamma=\sqrt{s_{N N}} /\left(2 m_{N}\right)$, (vi) Lorentz factor between both colliding-hadron rest frames, $\gamma=s_{N N} /\left(2 m_{N}^{2}\right)$, (vii) inverse of the colliding-hadron minimum impact parameter (giving the typical maximum photon cloud energy in the emitter rest frame), (viii) photon "cutoff energy" in the target or projectile rest frame, $E_{\gamma}^{\mathrm{B} \text { rest }}$ or $E_{\gamma \text { max }}^{\mathrm{A} \text { rest }}$, (ix) "maximum" photon-nucleon cms energy where $A$ or $B$ is the photon emitter, $\sqrt{s_{\gamma_{N}}^{\max }}$ or $\sqrt{s_{N \gamma}^{\max }},(\mathrm{x})$ photon "cutoff energy" in the cms, $E_{\gamma \text { max }}^{\mathrm{cms}}$, with both $A$ and $B$ emitting a photon coherently, (xi) "maximum" photon-photon $\mathrm{cms}, \sqrt{s_{\gamma \gamma}^{\max }}$.

Integrating eq. (2.1) over $b$, one has [22]

$$
\begin{aligned}
\frac{d n}{d k}=\frac{2 Z^{2} \alpha_{\mathrm{em}}}{\pi k}[ & \omega\left(b_{\min }, k\right) K_{0}\left(\omega\left(b_{\min }, k\right)\right) K_{1}\left(\omega\left(b_{\min }, k\right)\right) \\
& \left.-\frac{\omega\left(b_{\min }, k\right)^{2}}{2}\left(K_{1}^{2}\left(\omega\left(b_{\min }, k\right)\right)-K_{0}^{2}\left(\omega\left(b_{\min }, k\right)\right)\right)\right] .
\end{aligned}
$$

To avoid any confusion with the choice of the frame, it is useful to work with the momentum fraction or light cone coordinate, $x_{\gamma}=k / p_{h} \simeq k /\left(\gamma M_{N}\right)$, where $p_{h}$ is the momentum of the hadron emitting the photon. One trivially obtains

$$
\frac{d n}{d x_{\gamma}}=\left.\frac{k}{x_{\gamma}} \frac{d n}{d k}\right|_{\omega_{p A}=x_{\gamma} M_{p} b_{\min }} .
$$

The relation between the (differential) hadron-hadron cross section, $(d) \sigma^{h_{A} h_{B}}$, and the (differential) cross section for a photo-hadron scattering $\left(h_{A}\right.$ or $\left.h_{B}\right),(d) \sigma^{\gamma h_{A, B}}$, is naturally

\footnotetext{
${ }^{3}$ For Arp and O $p$ luminosity with AFTER@LHC, we conservatively assumed the same extracted flux of $\mathrm{Ar}$ and $\mathrm{O}$ as for $\mathrm{Pb}$, i.e. $2 \times 10^{5} \mathrm{~Pb} / \mathrm{s}$. See also [6, 23-25].
} 


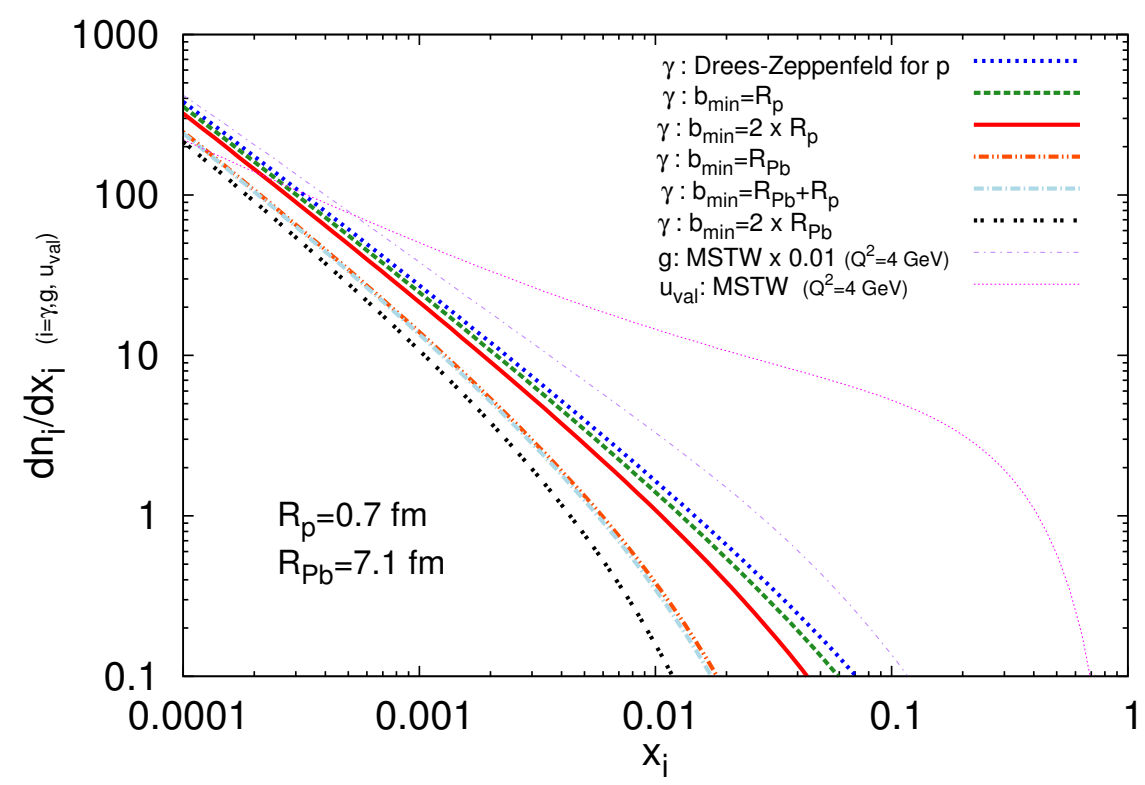

Figure 1. $\frac{d n}{d x}$ for photons from protons (blue dot for Drees-Zeppenfeld, green dash for $b_{\min }=R_{p}$ and red solid for $b_{\min }=2 R_{p}$ ) and from $\mathrm{Pb}$ (orange double dot dash for $b_{\min }=R_{\mathrm{Pb}}$, light blue dot dash for $b_{\min }=R_{\mathrm{Pb}}+R_{p}$ and black double dot for $\left.b_{\min }=2 R_{\mathrm{Pb}}\right)$ divided by $Z_{\mathrm{Pb}}^{2}$. These are compared to the gluon (thin pink long-dash, divided by 100) and $u$ quark (thin purple dot-dash) MSTW PDF in the proton.

given by the following convolution with the photon flux

$$
\begin{aligned}
d \sigma^{h_{A} h_{B}} & =\int d k_{\gamma}\left(\frac{d n^{h_{A}}}{d k_{\gamma}} d \sigma^{\gamma h_{B}}\left(s_{\gamma h_{B}}\left(k_{\gamma}\right)\right)+\frac{d n^{h_{B}}}{d k_{\gamma}} d \sigma^{\gamma h_{A}}\left(s_{\gamma h_{A}}\left(k_{\gamma}\right)\right)\right) \\
& =\int d x_{\gamma}\left(\frac{d n^{h_{A}}}{d x_{\gamma}} d \sigma^{\gamma h_{B}}\left(s_{\gamma h_{B}}\left(k_{\gamma}\left(x_{\gamma}\right)\right)+\frac{d n^{h_{B}}}{d x_{\gamma}} d \sigma^{\gamma h_{A}}\left(s_{\gamma h_{A}}\left(k_{\gamma}\left(x_{\gamma}\right)\right)\right)\right) .\right.
\end{aligned}
$$

By analogy with the parton model formulae, one can thus write:

$$
\varphi_{\gamma}\left(x_{\gamma}\right)=\frac{d n}{d x_{\gamma}}
$$

and interpret the latter as an equivalent-photon PDFs off the hadron (or the ions) by leaving the emitter intact.

In the case where the emitter is a proton, one could think that it is more accurate to take into account the spatial distribution of the charges through a form factor, which leads [27] to

$$
\frac{d n}{d x_{\gamma}}=\frac{\alpha}{2 \pi x_{\gamma}}\left(1+\left(1-x_{\gamma}\right)^{2}\right)\left[\ln c-\frac{11}{6}+\frac{3}{c}-\frac{3}{2 c^{2}}+\frac{1}{3 c^{2}}\right]
$$

where $c=1+\frac{0.71 \mathrm{GeV}^{2}}{Q_{\min }^{2}}$, and $Q_{\min }^{2}=M_{p}^{2} x_{\gamma}^{2}$. This is only strictly relevant for $e p$ collisions and at rather large $x_{\gamma}$. In $p p$ or $p A$ collisions, one indeed needs to take into account the radius of the other colliding object. The impact parameter $b$ is therefore not getting close to $R_{p}$. We will refer to this choice by "Drees-Zeppenfeld". 
Figure 1 shows a comparison (up to the charge factor $Z^{2}$ ) between the equivalent flux from a proton and from a lead ion. For $x_{\gamma}<10^{-3}$, the spectra show a similar behaviour, with a magnitude differing by less than a factor 2 . At larger $x_{\gamma}$, the proton spectrum is clearly harder because of the smaller proton size. This is the expected behaviour.

One could also consider the case where the proton emitting the photon breaks apart. In this situation, the photon is in fact radiated by the quarks and the corresponding photon PDF can then approximated by [28]

$$
\varphi_{\gamma}^{\text {break-up }}\left(x, Q^{2}\right)=\frac{\alpha}{2 \pi} \log \frac{Q^{2}}{Q_{0}^{2}} \sum_{q} \int_{x}^{1} \frac{d y}{y} P_{\gamma q}(x / y)\left[q\left(y, Q^{2}\right)+\bar{q}\left(y, Q^{2}\right)\right],
$$

with $P_{\gamma q}(z)=e_{q}^{2}\left(1+(1-z)^{2}\right) / z, Q_{0}^{2}$ is an energy cut-off, and $q\left(x, Q^{2}\right)$ is the quark PDFs in the proton. We will not consider this possibility further in this study, although such a process could contribute to the semi-exclusive case with still a large rapidity gap between the dilepton and the proton emitter. Another possibility, which we will discuss in section 5 is to have an elastic hadronic reaction via the exchange of a pomeron (or possibly an odderon).

\subsection{Fluxes and the rapidity dependence of the produced particles}

\subsubsection{Single-photon case}

If we consider the total cross section, $\sigma^{\gamma h}$, to photo-produce a particle of mass $Q$, we note that it can only be function of $s_{\gamma h}$ and $Q^{2}$ since it is already integrated on the final state variables. However, nothing prevents us to perform a change of variable in eq. (2.4) from $k_{\gamma}$ to a final state variable of the $\gamma$-hadron process, for instance the rapidity $y$ of the produced particle, keeping the other fixed. ${ }^{4}$ By momentum conservation, $y$ would enter ${ }^{5}$ via $k_{\gamma}(y)$ in $s_{\gamma h}$

Indeed, in our case, it is clearly instructive, in order to understand where the produced particle by photon-induced collisions fly, to consider the flux as a function of the particle rapidity $y$. For instance, we anticipate [6] that rapidities (in the hadron-hadron cms frame) from -4 to +1 should be accessible. A detector like LHCb, covering pseudorapidities from 2 to 5 would for instance approximately cover cms rapidities from -2.8 to 0.2 (see later the discussion about the acceptance).

For fixed $q_{T}$ and $Q^{2}, k_{\gamma}(y)$ is also fixed. From eq. (2.4), one trivially obtains

$$
\begin{aligned}
d \sigma^{h h} & =\int d k_{\gamma}\left(\frac{d n^{h_{A}}}{d k \gamma} d \sigma^{\gamma h_{B}}\left(s_{\gamma h_{B}}\left(k_{\gamma}\right)\right)+\frac{d n^{h_{B}}}{d k \gamma} d \sigma^{\gamma h_{A}}\left(s_{\gamma h_{A}}\left(k_{\gamma}\right)\right)\right) \\
& =\int d y\left(\frac{d n^{h_{A}}}{d y} d \sigma^{\gamma h_{B}}\left(s_{\gamma h_{B}}^{A}(y)\right)+\frac{d n^{h_{B}}}{d y} d \sigma^{\gamma h_{A}}\left(s_{\gamma h_{A}}^{B}(y)\right)\right)
\end{aligned}
$$

where $s_{\gamma h_{(B, A)}^{(A, B)}}^{(y)} \equiv s_{\gamma h_{(B, A)}}\left(k_{\gamma \text { from }(\mathrm{A}, \mathrm{B})}(y)\right)$ and therefore

$$
\frac{d \sigma^{h h}}{d y}=\frac{d n^{h_{A}}}{d y} d \sigma^{\gamma h_{B}}\left(s_{\gamma h_{B}}^{A}(y)\right)+\frac{d n^{h_{B}}}{d y} d \sigma^{\gamma h_{A}}\left(s_{\gamma h_{A}}^{B}(y)\right) .
$$

\footnotetext{
${ }^{4}$ In the following, we denote the 4-momentum of this particle $q=\left(q_{0}, \vec{q}_{T}, q_{z}\right)$ and $q_{T}=\left|\vec{q}_{T}\right|$.

${ }^{5}$ Defining $x_{\gamma}=s_{\gamma h} / s$, one indeed gets $x_{\gamma}=x_{\gamma}\left(y, q_{T}^{2}, Q, \epsilon\right)$ where $\epsilon= \pm 1$ when the projectile (target) is the photon emitter. See the appendix A for details.
} 


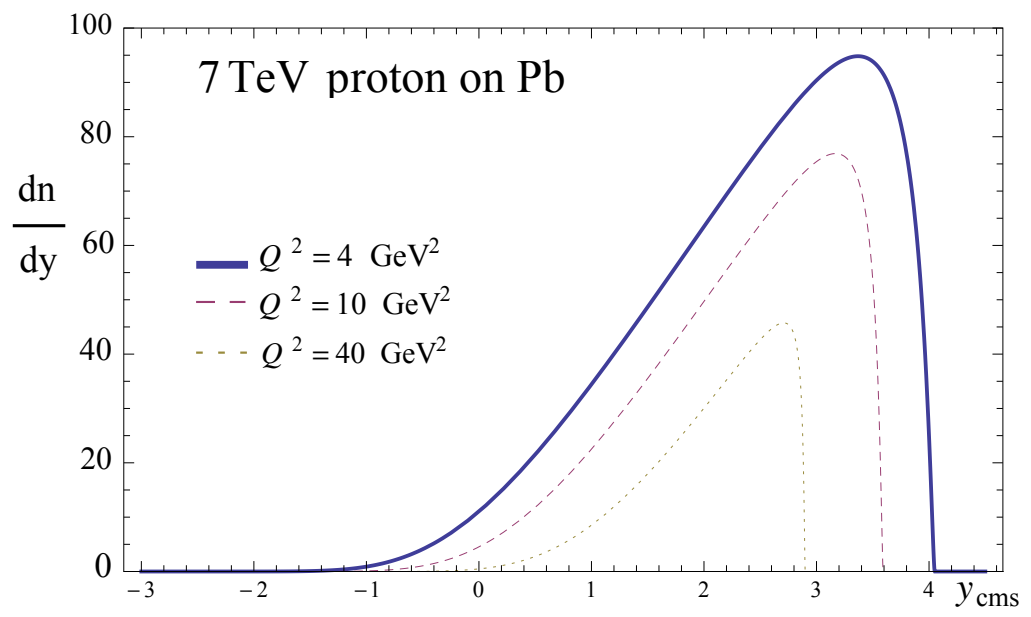

(a) proton on $\mathrm{Pb}$.

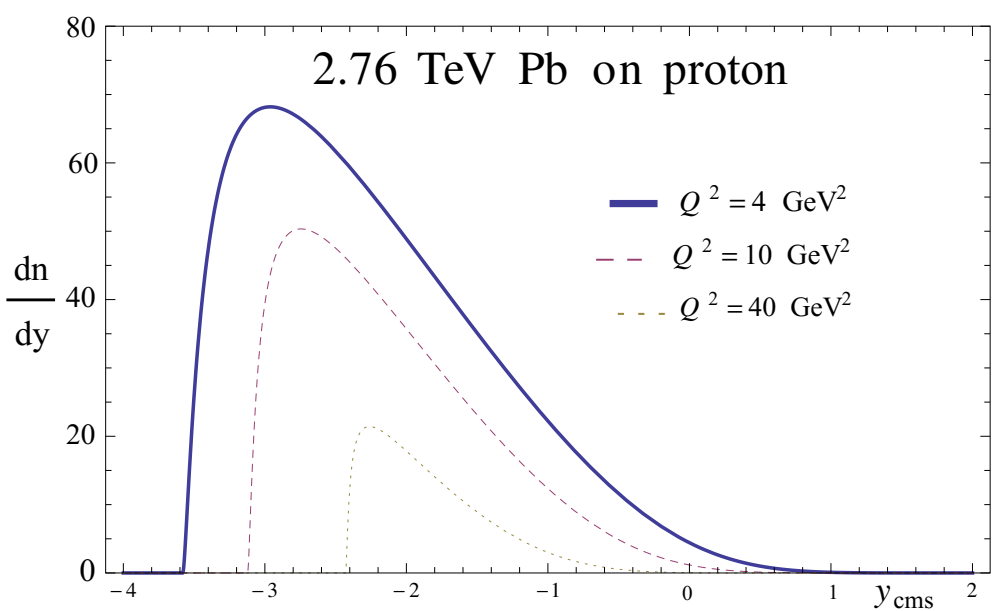

(b) $\mathrm{Pb}$ on $\mathrm{H}$.

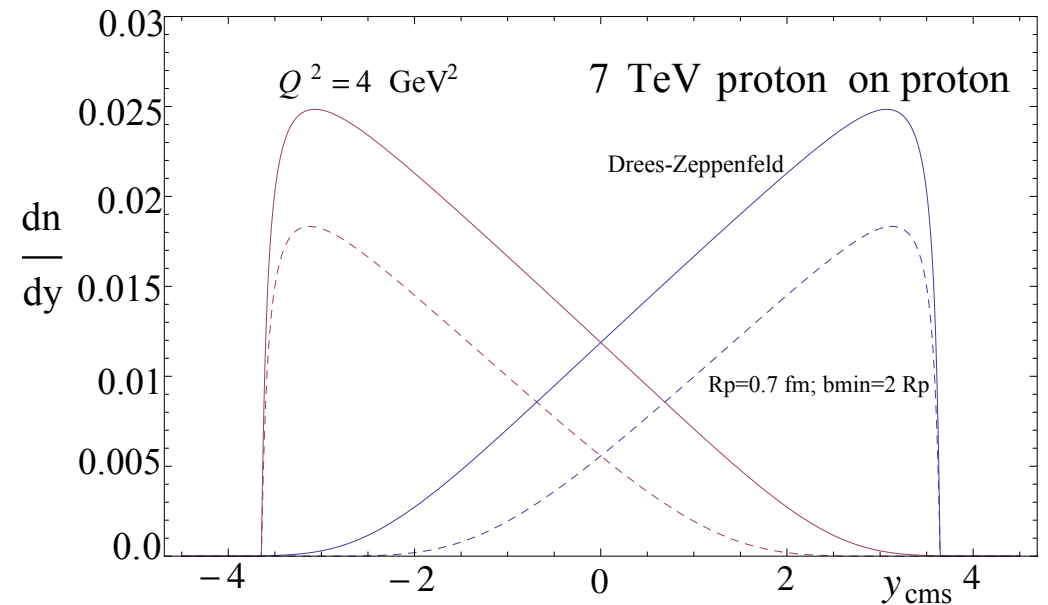

(c) proton on $\mathrm{H}$.

Figure 2. $\frac{d n}{d y}$ for the proton run on $\mathrm{Pb}$ nucleon target (a), and for $\mathrm{Pb}$ run on $\mathrm{H}$ target $(\mathrm{b})$, and (c) for p-p run. The flux is calculated for the specified $Q^{2}$ at $q_{T}=0$ (e.g. $t=t_{0}$ ). 
We therefore find it instructive to plot $\frac{d n}{d y}$ (for fixed $q_{T}$ and $Q^{2}$ ) for different configurations: figure 2a shows the case of $7 \mathrm{TeV}$ protons on lead, the lead being the photon emitter; figure $2 \mathrm{~b}$ shows the case of $2.76 \mathrm{TeV}$ lead on protons, the lead being the photon emitter; ${ }^{6}$ figure $2 \mathrm{c}$ shows the case of $7 \mathrm{TeV}$ protons on proton (i.e. hydrogen), where both can be the photon emitter (note that the corresponding fluxes cannot simply be summed).

In the first case (figure 2a), the flux is maximum in the forward region, which corresponds to a soft (coherent) emission by the lead target. In general, the particle tends to be emitted in the "hadron-receiver" region since the photon momentum is very small (here $y_{\text {receiver=beam }}=4.8$ ). To be more precise, the boost - or rapidity difference $\Delta y$ - between the photon-hadron and hadron-hadron cms, $\Delta y_{h_{A} h_{B}}^{\gamma h_{A, B}}=y_{\mathrm{cms}}^{h_{A} h_{B}}-y_{\mathrm{cms}}^{\gamma h_{A, B}}$, is simply related to the photon momentum fraction via

$$
\Delta y_{h_{A} h_{B}}^{\gamma h_{A, B}}=-\epsilon \frac{1}{2} \ln x_{\gamma},
$$

In order to produce a particle of mass $\mathrm{Q}$ at threshold ${ }^{7}$ in a photon-hadron collision at $\sqrt{{ }_{\gamma h}}$ resulting from a hadron-hadron UPC at $\sqrt{s_{N N}}$, one approximately has $x_{\gamma} \simeq Q^{2} / s_{N N}$. At AFTER@LHC, for $Q^{2}=4 \mathrm{GeV}^{2}, x_{\gamma}=3 \times 10^{-4}$, which gives $\Delta y_{h_{A} h_{B}}^{\gamma h_{A, B}} \simeq-4$. This explains the maximum at $y_{\mathrm{cms}} \simeq 4$ of the solid line of figure $2 \mathrm{a}$.

If the emission is too soft, there is simply not enough energy to create a particle of a given mass, $Q$, - this explains why the curve for $Q^{2}=4(40) \mathrm{GeV}^{2}$ drops sharply at $y_{\mathrm{cms}} \simeq 4(3)$. In the second case (figure $2 \mathrm{~b}$ ), the flux is the highest in the opposite direction where soft photons are emitted by the lead projectile. Since the lead beam energy is lower $\left(y_{\text {beam }}=4.2\right)$ and the energy cut-off smaller, the particle is less backward than it is forward in the first case. In the third case (figure 2c), both proton can emit. The behaviour is similar to (a) and (b), but for a lower value due to the absence of the factor $Z^{2}$. It is however harder in the forward (backward) region for a projectile (target) proton emitter - note that the flux in the tail is still nonzero at $y$ down to \pm 3 . This mirrors the possibility for harder photon (up to $x_{\gamma} \simeq 0.1$ ) emission from a proton compared to a larger nucleus. The solid and dashed curves on figure $2 \mathrm{c}$ refer to two different fluxes: Drees-Zeppenfeld as in $e p$ collisions (solid) and $b_{\min }=2 \times R_{p}$ (dashed).

The advantage offered by the fixed-target mode is therefore obvious when the emitter is the projectile. In such a case, the large rapidity differences between the photon-hadron and hadron-hadron cms and that between the hadron-hadron cms and the laboratory frame nearly cancel. This results in the production of the particle at slightly positive rapidities which are easily covered by typical detectors.

\subsubsection{Double-photon case}

Obviously, one can also consider UPC where both colliding hadrons radiate a photon. This is expected to be the dominant reaction at work in dilepton production, $A A^{\prime} \rightarrow A \ell^{+} \ell^{-} A^{\prime}$,

\footnotetext{
${ }^{6}$ In this case, as for (a), the photon emission by a lead ion can, in principle, be tagged with a neutron emission. In addition, the probability for the proton to be the emitter is, in practice, negligible $\left(Z^{2}\right.$ suppressed at the same $\left.\left|y_{c m s}\right|\right)$. We therefore do not show the curve for this possibility.

${ }^{7}$ I.e. at rest in the photon-hadron $\mathrm{cms}$.
} 


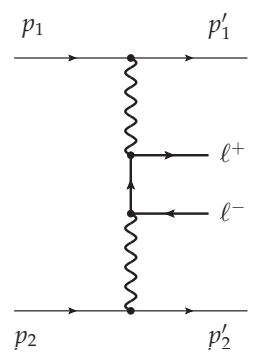

(a) $\mathrm{BH}$.

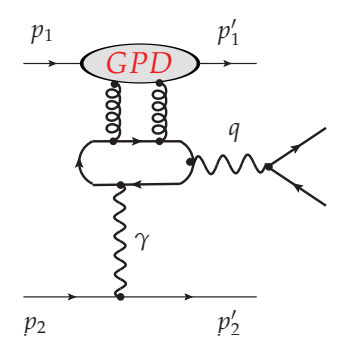

(b) TCS with gluon GPDs.

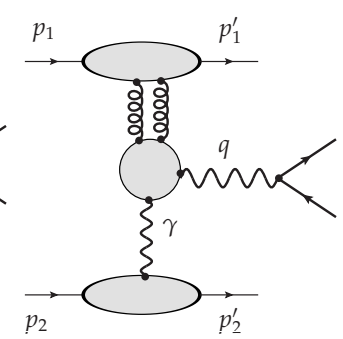

(c) $\gamma \mathbb{P}$ fusion.

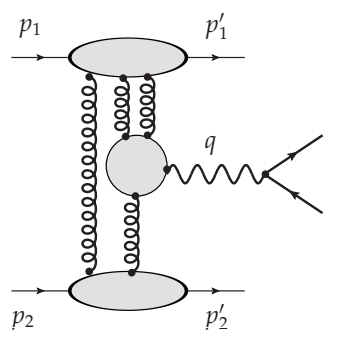

(d) $\mathbb{O P}$ fusion.

Figure 3. Feynman graphs representing the scattering of two hadrons of momentum $p_{1}$ and $p_{2}$ remaining intact with final state momenta $p_{1}^{\prime}$ and $p_{2}^{\prime}$ producing a lepton pair via (a) the $\mathrm{BH}$ process by photon-photon fusion, (b) via TCS described in terms of a GPD (here the gluon one), (c) via photon-pomeron $(\gamma \mathbb{P})$ fusion represented by the minimal number of gluon exchanges and (d) via odderon-pomeron $(\mathbb{O P})$ fusion also represented with the minimal number of gluon exchanges.

$\mathrm{PbH} \rightarrow \mathrm{Pb} \ell^{+} \ell^{-} \mathrm{H}, p \mathrm{~Pb} \rightarrow p \ell^{+} \ell^{-} \mathrm{Pb}$ or $p p^{\prime} \rightarrow p \ell^{+} \ell^{-} p^{\prime}$, via $\gamma \gamma \rightarrow \ell^{+} \ell^{-}$(see figure $3 \mathrm{a}$ ), that is the $\mathrm{BH}$ process. Combining the fluxes from both hadrons, one can derive a joint photon flux or $\gamma \gamma$ luminosity as function of their invariant mass, $\sqrt{s_{\gamma \gamma}}$ or $W$, and rapidity $Y$, as it is usually done for $g g$ luminosity at the LHC to discuss $H^{0}$ production rates, for instance.

Figure 5 shows the rapidity dependence of $\gamma \gamma$ luminosity in the hadron-hadron cms at a fixed $W$, obtained from of eqs. 42, 43, 50, 51 and 52 of [29] with numerical integrations on the impact parameters. In the case of the $\mathrm{BH}$ process discussed in detail in the next section, the invariant mass of the pair is that of the dilepton. One observes that the maxima in the $\mathrm{Pb} p$ and $p \mathrm{~Pb}$ fluxes occur at respectively backward (forward) rapidities because the photon spectrum from the proton is harder than that from the ion.

\section{Lepton-pair production: energy, invariant-mass, rapidity and transverse- momentum dependencies}

We now discuss in more details the dominant process involved in lepton-pair production that is $\mathrm{BH}$ from $\gamma \gamma \rightarrow \ell^{+} \ell^{-}$(figure $3 \mathrm{a}$ ) in hadron-hadron collisions.

\subsection{Total cross section and for fixed $Q$}

The total cross section for dimuon production by two heavy and charged particles is well known and can readily be computed from the analytical formula of Racah [30]. At $\sqrt{s}=115 \mathrm{GeV}$, one gets $15.0 \mathrm{nb}$ for $p p$ collisions, to be compared with $16.5 \mathrm{nb}$ with the Starlight Monte Carlo code. ${ }^{8}$ The corresponding cross section for $p \mathrm{~Pb}$ is simply obtained by multiplying by $Z^{2}$, i.e. $100 \mu \mathrm{b}$. In this case, it is assumed that the particle are point-like. STARLIGHT rather gives $36 \mu \mathrm{b}$.

We are however interested in the differential cross sections for particular values of the dilepton invariant mass, $Q$; muon pairs produced at $Q \gtrsim 2 m_{\mu}$ are usually difficult to study

\footnotetext{
${ }^{8}$ STARLIGHT website.
} 


\begin{tabular}{|lc|cc|cc|cc|c|}
\hline System & \multicolumn{2}{c}{$p p$} & \multicolumn{2}{c}{$p \mathrm{~Pb}$} & \multicolumn{2}{c}{$\mathrm{Pb} p$} & \multicolumn{2}{c|}{$\mathrm{PbPb}$} \\
\hline$\sqrt{s_{N N}}[\mathrm{GeV}]$ & \multicolumn{2}{c}{115} & \multicolumn{2}{c}{115} & \multicolumn{2}{c|}{72} & \multicolumn{2}{c|}{72} \\
& $\mathrm{BW}$ & $\mathrm{SL}$ & $\mathrm{BW}$ & $\mathrm{SL}$ & $\mathrm{BW}$ & $\mathrm{SL}$ & $\mathrm{BW}$ & $\mathrm{S}$ \\
$\frac{d \sigma_{\ell \ell}(Q=2 \mathrm{GeV})}{d Q}[\mathrm{nb} / \mathrm{GeV}]$ & 0.14 & 0.15 & 200 & 210 & 77 & 84 & 7000 & 7100 \\
$\frac{d \sigma_{\ell \ell}\left(Q=2 \mathrm{GeV}, y_{\mathrm{cms}}^{++}=0\right)}{d Q d \ell_{\mathrm{cms}}^{\ell \ell}}[\mathrm{nb} / \mathrm{GeV}]$ & 0.039 & 0.038 & 39 & 45 & 14 & 16 & 5500 & 5600 \\
$\frac{d \sigma_{\ell \ell}(Q=2.98 \mathrm{GeV})}{d Q}[\mathrm{nb} / \mathrm{GeV}]$ & 0.03 & 0.031 & 32 & 34 & 9.7 & 11 & 230 & 250 \\
$\frac{d \sigma_{\ell \ell}\left(Q=2.98 \mathrm{GeV}, y_{\mathrm{cms}}^{\ell^{+}}=0\right)}{d Q d y_{\mathrm{cms}}^{\ell \ell}}[\mathrm{nb} / \mathrm{GeV}]$ & 0.009 & 0.009 & 5.7 & 6.5 & 1.3 & 1.6 & 200 & 210 \\
\hline
\end{tabular}

Table 2. BH differential cross section for fixed dilepton masses integrated (or not) on $y_{\mathrm{cms}}^{\ell \ell}$. "BW" denotes Breit-Wheeler and "SL" denotes STARLIGHT.

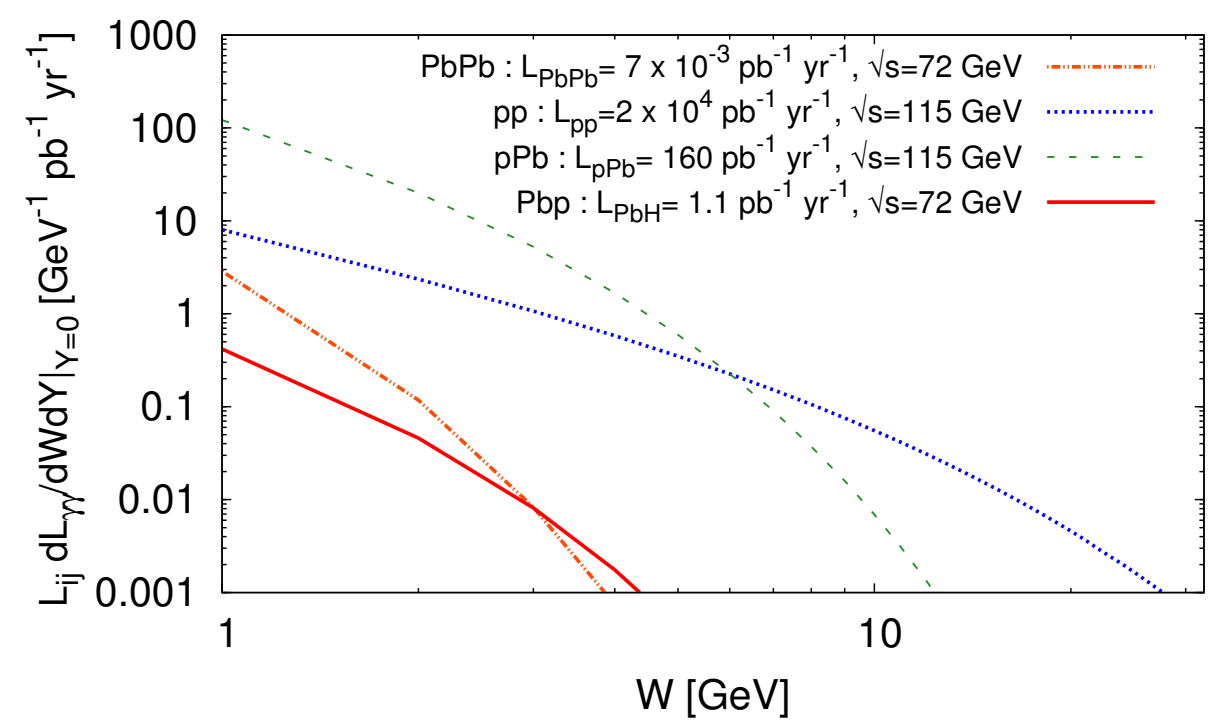

Figure 4. $\mathcal{L}_{i j} \times \frac{d L_{\gamma \gamma}}{d W}$ from protons and from $\mathrm{Pb}$ in $p p, p \mathrm{~Pb}, \mathrm{PbH}$ and $\mathrm{PbPb}$ collisions (see table 1) as a function of the invariant mass of the photon pair, $W$, at $Y_{\mathrm{cms}}=0$ of the $\gamma \gamma$ pair (or of the to-be produced dilepton).

since the muons have small transverse momenta. Such a cross section can easily be obtained by combining two EPA photon fluxes as done to obtain $\frac{d L_{\gamma \gamma}}{d W d Y}$ and then by integrating over $Y$. One then convolve it with the Breit-Wheeler formula [31]:

$$
\sigma_{\ell \ell}^{\gamma \gamma}\left(Q^{2}\right)=\frac{4 \pi \alpha^{2}}{Q^{2}}\left[\left(2+\frac{8 m_{\ell}^{2}}{Q^{2}}-\frac{16 m_{\ell}^{4}}{Q^{4}}\right) \ln \frac{Q+\sqrt{Q^{2}-4 m_{\ell}^{2}}}{2 m_{\ell}}-\sqrt{1-\frac{4 m_{\ell}^{2}}{Q^{2}}}\left(1+\frac{4 m_{\ell}^{2}}{Q^{2}}\right)\right] .
$$

We have checked that we obtained the same result as STARLIGHT for dimuon production, for instance for $Q=(2,2.98) \mathrm{GeV}$ for $p p$ and $p \mathrm{~Pb}$ collisions at $\sqrt{s_{N N}}=115 \mathrm{GeV}$, as well as for $\mathrm{Pb} p$ and $\mathrm{PbPb}$ collisions at $\sqrt{s_{N N}}=72 \mathrm{GeV}$, up to the uncertainty attached to the value taken for the nucleus radius, see the first and third rows of results on table 2 .

\footnotetext{
${ }^{9}$ The reason why we took $W=m_{\eta_{c}}=2.98 \mathrm{GeV}$ for this example will become clear in the next section.
} 


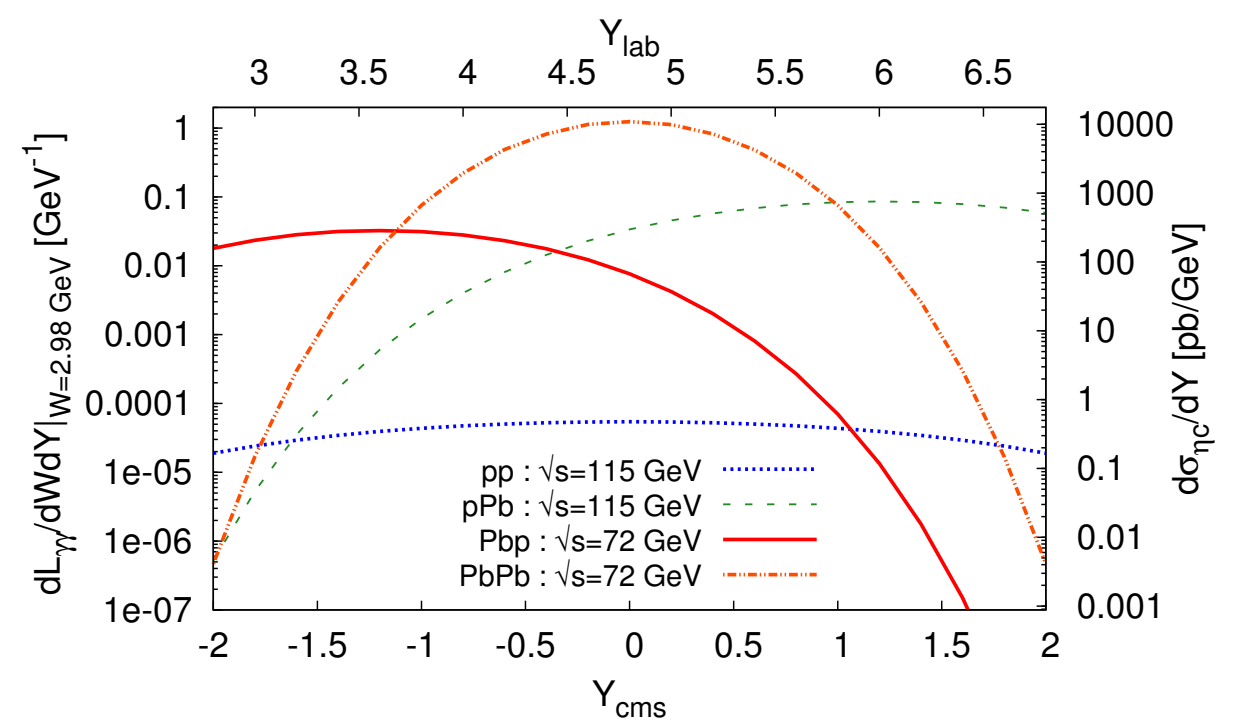

Figure 5. $\left.\frac{d L_{\gamma \gamma}}{d W d Y}\right|_{W=m_{\eta_{c}}}$ (and $\frac{d \sigma_{\eta_{c}}}{d Y}$ ) as a function of the rapidity, in the hadron-hadron cms (lower $x$-axis) and in the laboratory frame (upper $x$-axis). ${ }^{9}$

One readily obtains the rapidity dependence of the differential cross section, by simply using $\left.\frac{d L_{\gamma \gamma}}{d W d Y}\right|_{W=Q, Y=y_{\mathrm{cms}}^{\ell \ell}}$. We already discussed it on figure 5. The $\gamma \gamma$ luminosity at a fixed cms rapidity of the photon pair, $Y_{\mathrm{cms}}$ (or equivalently $y_{\mathrm{cms}}^{\ell \ell}$ ), is plotted as a function of $W$ on figure 4 namely at $Y_{\mathrm{cms}}=0$ for $p p, p \mathrm{~Pb}, \mathrm{~Pb} p$ and $\mathrm{PbPb}$ collisions; it is multiplied by the corresponding hadron yearly luminosity denoted $\mathcal{L}_{i j}$. Just as for the rapidity integrated results, both methods agree for $y_{\mathrm{cms}}^{\ell \ell}=0$ as it should be, see the second and fourth rows of results on table 2 .

Knowing $\frac{d L_{\gamma \gamma}}{d W d Y}$, one can also obtain the production cross section for a scalar or tensor quarkonium, $\mathcal{Q}$ provided that we know its partial width into a photon pair, $\Gamma_{\gamma \gamma}$ :

$$
\left.\frac{d \sigma_{\mathcal{Q}}^{h_{A} h_{B}}}{d Y_{\mathcal{Q}}} \stackrel{\gamma \gamma \rightarrow \mathcal{Q}}{=} 8 \pi^{2}\left(2 J_{\mathcal{Q}}+1\right) \frac{\Gamma_{\gamma \gamma}}{2 M_{\mathcal{Q}}^{2}} \frac{d L_{\gamma \gamma}}{d W d Y}\right|_{W=M_{\mathcal{Q}}, Y=Y_{\mathcal{Q}}}
$$

From $\Gamma_{\gamma \gamma}^{\eta_{c}}=5.1 \times 10^{-6} \mathrm{GeV}$ and $\Gamma_{\gamma \gamma}^{\chi_{c 2}}=5.3 \times 10^{-7} \mathrm{GeV}$ [32], one gets $\sigma_{\gamma \gamma}^{\eta_{c}}=8.8 \mathrm{nb}$ and $\sigma_{\gamma \gamma}^{\chi_{c 2}}=3.2 \mathrm{nb}$. The rapidity dependence of this cross section is thus up to a constant factor that of the joint flux at the corresponding ${ }^{10} W$ (see figure 5). In particular, for $p p(p \mathrm{~Pb})$ collisions at $\sqrt{s_{N N}}=115 \mathrm{GeV}$, the $\eta_{c}$ cross sections at $y=0$ in the hadron-hadron cms are $0.5 \mathrm{pb}(0.4 \mathrm{nb})$ and for $\mathrm{Pb} p(\mathrm{PbPb})$ collisions at $\sqrt{s_{N N}}=72 \mathrm{GeV}, 67 \mathrm{pb}(11 \mathrm{nb})$. With the yearly luminosities in table 1 , one can respectively expect $10^{4}, 1.8 \times 10^{6}, 74$ and $80 \eta_{c}$ per year.

At this point, two remarks are in order. First, we stress that although, $m_{\eta_{c}}$ is above the energy "cut-off" showed in table 1 for the systems $p \mathrm{~Pb}, \mathrm{~Pb} p$ and $\mathrm{PbPb}$, the rates are nonzero using realistic photon fluxes. Second, our result for $p p$ collisions is 2-3 times below

\footnotetext{
${ }^{10}$ Hence our choice of $W=m_{\eta_{c}}=2.98 \mathrm{GeV}$ in figure 4.
} 


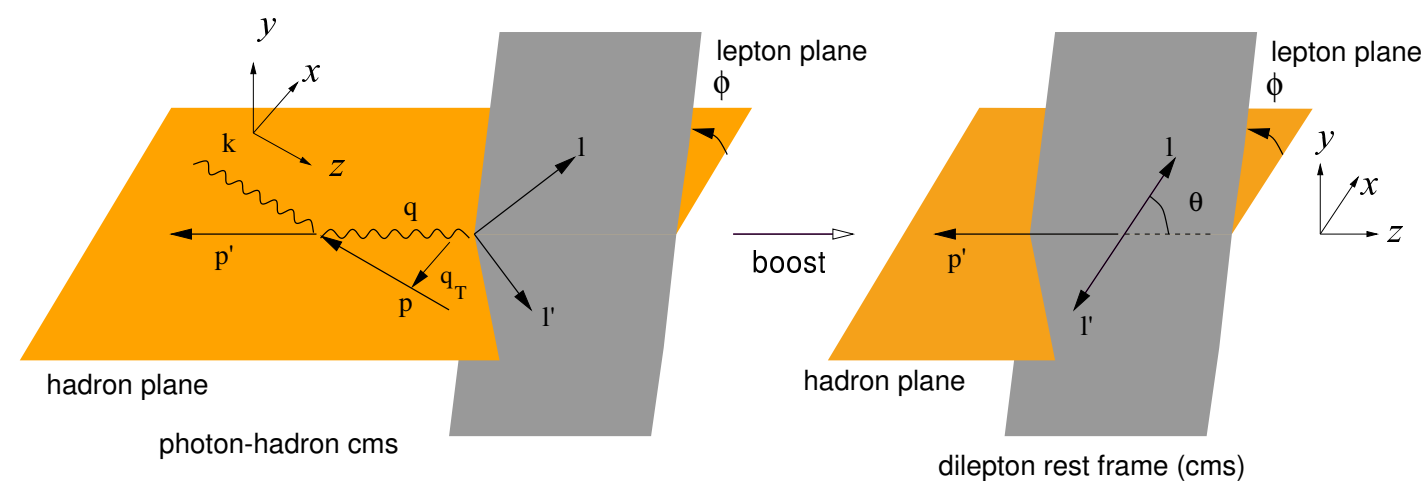

Figure 6. Definition of the kinematical variables in $\gamma(k) h(p) \rightarrow \ell^{+}\left(l^{\prime}\right) \ell^{-}(l) p\left(p^{\prime}\right)$ in particular the angles $\theta$ and $\phi$ attached to the $\ell^{-}$.

that recently derived by Goncalves and Sauter [33]. We attribute this difference to the fact that they used the Drees and Zeppenfeld photon flux, which is precisely twice larger than that derived with $b>2 R_{p}$ at $x_{\gamma} \simeq \sqrt{s} / m_{\eta_{c}} \simeq 0.025$ (see figure 1 ). As we noted above, the former should only strictly be used for $e p$ collisions. Such a difference only arises at 'low' hadron-hadron cms energies which correspond to $x_{\gamma}$ on the order of $10^{-2}$ and above. A measurement of the $\eta_{c}$ or $\chi_{c 2}$ cross section in exclusive $p p$ collisions at AFTER@LHC is therefore ideal to tell which choice is the most appropriate.

\subsection{Production at nonzero transverse momenta}

If we prefer to look at dileptons produced in reactions characterised by a momentum transfer squared of one emitter, $|t|$, up to $1 \mathrm{GeV}^{2}$, which results in a measurable transverse momentum, $q_{T}$, of the dilepton, it may be more suitable to treat the photon emission using a nucleon form factor. ${ }^{11}$ By construction, the off-shellness of this photon cannot be neglected. This amounts to consider the process photoproduction, $\gamma p \rightarrow \ell^{+} \ell^{-} p$, where the beam ${ }^{12}$ photon flux is still treated in the EPA approximation and the effect of the form factor embedded in the photoproduction cross section.

Following [8], this $\gamma h$ differential cross section for an unpolarised target to be convoluted with the beam photon flux (provided that $\sin \theta \gg m_{\ell} / Q$ ) reads

$$
\begin{aligned}
& \frac{d \sigma_{\ell \ell}^{\gamma h \mathrm{BH}}}{d Q^{2} d q_{T}^{2} d \cos \theta d \phi}\left(y_{\mathrm{cms}}^{\ell \ell}\right) \approx J \frac{\alpha_{e m}^{3}}{2 \pi s_{\gamma h}^{2}} \frac{1}{-t} \frac{1+\cos ^{2} \theta}{\sin ^{2} \theta} \\
& \quad \times\left.\left[\left(F_{1}^{2}(t)-\frac{t}{4 M_{N}^{2}} F_{2}^{2}(t)\right) \frac{2\left(s_{\gamma h}-M_{N}^{2}\right)^{2}}{Q^{4}} \frac{q_{T}^{2}}{-t}+\left(F_{1}(t)+F_{2}(t)\right)^{2}\right]\right|_{t=t\left(y_{\mathrm{cms}}^{\ell \ell}, q_{T}^{2}, Q, \epsilon\right)},
\end{aligned}
$$

where $F_{1,2}$ are respectively the Dirac and Pauli form factors evaluated at $t=\left(p-p^{\prime}\right)^{2}=$ $(k-q)^{2}$, the quantity $\epsilon$ and procedure to obtain $t\left(y_{\mathrm{cms}}^{\ell \ell}, q_{T}^{2}, Q, \epsilon\right)$ and $J$ (the Jacobian to change from $t$ to $q_{T}^{2}$ ) are explained in the appendix A. The $\theta$ and $\phi$ angles are defined

\footnotetext{
${ }^{11}$ In the nuclear case, such a configuration is admittedly much suppressed.

${ }^{12}$ The term "beam" may be improper in the fixed-target case since this photon can very well be emitted by a nucleon or nucleus in the target; this is particularly true for $p \mathrm{~Pb}$ collisions.
} 
as on figure 6. The apparent divergence at $\theta \rightarrow 0$ is regulated when the lepton mass is kept, just as in the Breit-Wheeler equation, eq. (3.1), which is logarithmically divergent for $m_{\ell} \rightarrow 0$. We note that $\theta$ - the polar angle of the lepton - which is defined in the rest frame of the dilepton, can only be approximately related to the (pseudo)-rapidity of the lepton in the cms frame for $y_{\mathrm{cms}}^{(\ell)} \simeq 0$ and $q_{T} \ll Q$. In such a case, the particular configuration $\theta \rightarrow \pi / 2$ corresponds to $y_{\mathrm{cms}}^{\ell} \simeq \eta_{\mathrm{cms}}^{\ell} \simeq 0$ which falls into the acceptance of a detector like LHCb and ALICE (with Pb beams). Using eq. (2.9), one then obtains $d \sigma_{\ell \ell}^{h h \mathrm{BH}} /\left(d Q^{2} d q_{T}^{2} d \cos \theta d \phi d y_{\mathrm{cms}}^{\ell \ell}\right)$.

\section{Timelike Compton scattering or exclusive photoproduction of a dilep- ton}

The process of the lepton-pair production in ultraperipheral collisions may also be used to investigate hadron structure in terms of GPDs through the measurement of the contribution of the TCS process, figure 3b, to the cross section. Although the BH amplitude squared is much larger than the TCS one, it is possible to study the interference term between TCS and BH processes, which may be projected out through the analysis of the angular distribution of the produced leptons [8] and which depends on the GPDs. The interference term of the differential cross section is given by:

$$
\begin{aligned}
\frac{d \sigma_{\ell \ell}^{\gamma h \mathrm{INT}}}{d Q^{2} d t d \cos \theta d \phi} \approx & -\frac{\alpha_{e m}^{3}}{4 \pi s_{\gamma h}^{2}} \frac{\sqrt{t_{0}-t}}{-t Q} \frac{\sqrt{1-\eta^{2}}}{\eta}\left(\cos \phi \frac{1+\cos ^{2} \theta}{\sin \theta}\right) \\
& \times \operatorname{Re}\left[F_{1}(t) \mathcal{H}(\eta, t)-\eta\left(F_{1}(t)+F_{2}(t)\right) \tilde{\mathcal{H}}(\eta, t)-\frac{t}{4 M^{2}} F_{2}(t) \mathcal{E}(\eta, t)\right]
\end{aligned}
$$

where we have neglected lepton mass and assumed that $s_{\gamma h}, Q^{2} \gg t, M_{N}^{2}$. The variable $\eta$ called skewedness is given by:

$$
\eta=\frac{Q^{2}}{2 s_{\gamma h}-Q^{2}},
$$

and $t_{0}$ is maximal value of squared momentum transfer $t$ reached at $q_{T}=0$, and is equal $t_{0}=-4 M^{2} \frac{\eta^{2}}{1-\eta^{2}}$ up to corrections in $1 / Q^{2}$. The functions $\mathcal{H}(\eta, t), \tilde{\mathcal{H}}(\eta, t)$ and $\mathcal{E}(\eta, t)$ are the well known Compton form factors. These involve the GPD $H, \tilde{H}$ and $E$ (as defined in [17]) respectively through a convolution with the hard-scattering kernels $T_{\{H, \tilde{H}, E\}}^{q, g}$ computed at a given order in $\alpha_{s}$ :

$$
\begin{aligned}
& \{\mathcal{H}, \tilde{\mathcal{H}}, \mathcal{E}\}(\eta, t)= \\
& \int_{-1}^{1} d x\left[\sum_{q} T_{\{H, \tilde{H}, E\}}^{q}(x, \eta)\left\{H^{q}, \tilde{H}^{q}, E^{q}\right\}(x, \eta, t)+T_{\{H, \tilde{H}, E\}}^{g}(x, \eta)\left\{H^{g}, \tilde{H}^{g}, E^{g}\right\}(x, \eta, t)\right] .
\end{aligned}
$$

The expression for the kernels $T_{\{H, \tilde{H}, E\}}$ at $\mathrm{LO}$ are given in the appendix B. The Next-toLeading Order (NLO) hard-scattering kernel, ${ }^{13}$ which we use here, can be found in [10, 34].

\footnotetext{
${ }^{13}$ As done in [10], we set $\mu_{R}=\mu_{F}=Q$ in the hard-scattering kernel and, as for other similar phenomenological analyses, the GPDs are not evolved. In the case of the G-MSTW model, the GPDs can be made $\mu_{F}$ dependent through the evolution of the input PDFs.
} 

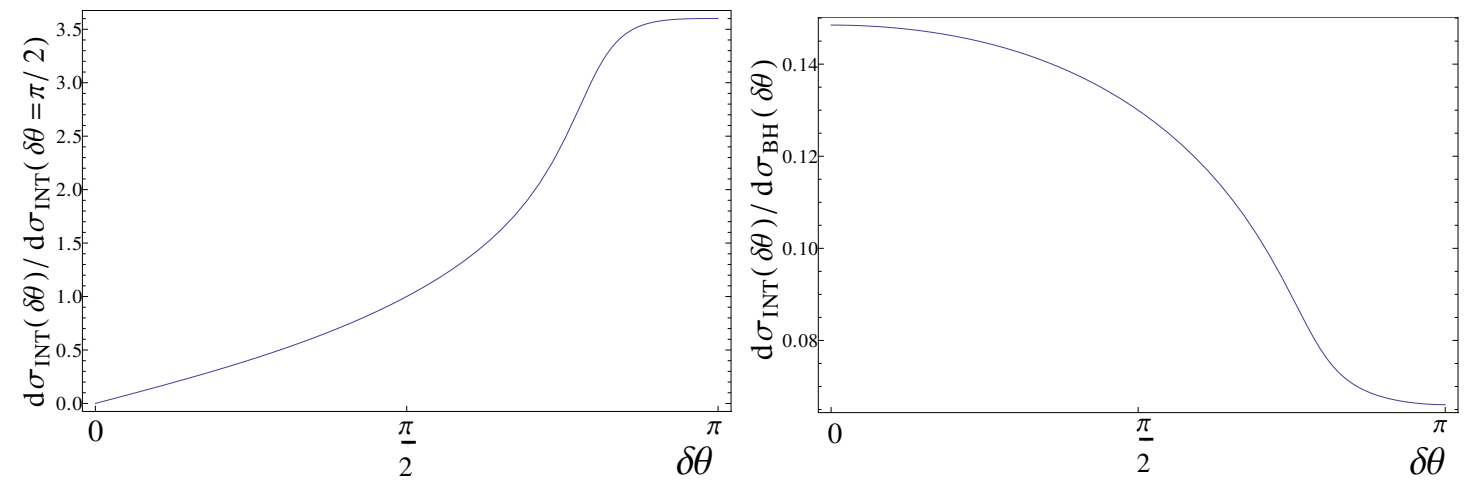

Figure 7. (left) $\frac{d \sigma_{\mathrm{INT}}}{d y}(\delta \theta) / \frac{d \sigma_{\mathrm{INT}}}{d y}(\delta \theta=\pi / 2)$, (right) $\frac{d \sigma_{\mathrm{INT}}}{d y} / \frac{d \sigma_{\mathrm{BH}}}{d y}(\delta \theta)$ for the model KG for $Q^{2}=$ $4 \mathrm{GeV}^{2}, t=-0.1 \mathrm{GeV}^{2}$, and $\phi=0$ for proton run on $\mathrm{Pb}$, integrated over $\theta \in(\pi / 4,3 \pi / 4)$ and for $y=0$ at NLO.

To advocate that the TCS measurement is feasible in the AFTER kinematics, we present phenomenological predictions making use of two GPD models through double distributions [15]: the first is based on the Goloskokov-Kroll (GK) model based on fits of meson electroproduction data and the second is a model using MSTW8 parton distribution function with a simple factorised $t$ dependence, referred to as G-MSTW (for a detailed description see [11]).

Comparing the $\phi$ dependence in eq. (3.3) to that in eq. (4.1), one directly sees that one can project out the interference term and eliminate the unwanted $\mathrm{BH}$ background by integrating the differential cross section over $\phi$ with the weight $\cos \phi$. To avoid the limit $\theta \rightarrow 0$ where the $\mathrm{BH}$ signal gets very large, one can integrate over $\theta$ symmetrically around $\pi / 2$ up to a value which depends on the size of the interference. Varying the limits of integration in $\theta$ according to

$$
\theta \in\left(\frac{\pi-\delta \theta}{2}, \frac{\pi+\delta \theta}{2}\right)
$$

results in the change of the strength of the interference BH-TCS with respect to the $\mathrm{BH}$ cross section shown on figure 7 . On the left panel, we show the ratio of the integral of the interference BH-TCS for a given $\delta \theta$ normalised to that for $\delta \theta=\pi / 2$. We see that for $\delta \theta$ close to $\pi$ the ratio of the integrals of the interference stops increasing. In any case, in this limit, as we present on the right panel, the ratio of the integral of the interference term to the $\mathrm{BH}$ one gets very small because the $\mathrm{BH}$ cross section gets large. ${ }^{14}$ In other words, there is no specific reason to take $\delta \theta$ much larger than $\pi / 2$; below this value the magnitude of the interference term is close to $10 \%$ of the $\mathrm{BH}$ term.

One can then combine the $\gamma h$ cross section with the photon flux as a function of the dilepton rapidity at a fixed $t$ to get the interference part of 5 -fold hadron-hadron differential cross section. Defining the integration region as

$$
\int_{\Omega} d^{5} F \equiv \int_{\pi / 4}^{3 \pi / 4} d \theta \int_{0}^{2 \pi} d \phi \int_{-2.5}^{0} d y \int_{0}^{0.25} d q_{T}^{2} \int_{1.5}^{3} d Q,
$$

\footnotetext{
${ }^{14}$ In one neglects the lepton mass, it even diverges.
} 
the $\mathrm{BH}$ cross section from a $7 \mathrm{TeV}$ proton beam on a $\mathrm{Pb}$ target is

$$
\sigma_{\mathrm{BH}}^{\mathrm{p} P b}=\int_{\Omega} d^{5} F \frac{d \sigma_{\mathrm{BH}}}{d Q d q_{T}^{2} d y d \theta d \phi}=1940 \mathrm{pb},
$$

which, for a luminosity of $0.16 \mathrm{fb}^{-1} \mathrm{yr}-1$, gives about $3 \cdot 10^{5}$ events per year. In the case of a $\mathrm{H}$ target, one has

$$
\sigma_{\mathrm{BH}}^{\mathrm{pH}}=\int_{\Omega} d^{5} F \frac{d \sigma_{\mathrm{BH}}}{d Q d q_{T}^{2} d y d \theta d \phi}=7.1 \mathrm{pb}
$$

which, for a luminosity $=20 \mathrm{fb}^{-1} \mathrm{yr}^{-1}$, gives $1.4 \cdot 10^{5}$ events per year for a $100 \mathrm{~cm}$ liquidhydrogen target. Finally, for $\mathrm{Pb}$ on $\mathrm{H}$, one has

$$
\sigma_{\mathrm{BH}}^{\mathrm{PbH}}=\int_{\Omega} d^{5} F \frac{d \sigma_{\mathrm{BH}}}{d Q d q_{T}^{2} d y d \theta d \phi}=5500 \mathrm{pb},
$$

which, for a luminosity $=11 \mathrm{nb}^{-1} \mathrm{yr}^{-1}$, gives $6 \cdot 10^{3}$ events per year for a $100 \mathrm{~cm}$ liquidhydrogen target. As aforementioned, we do not consider the case where the nucleus is emitter with a $|t|$ of a few hundred $\mathrm{MeV}^{2}$ which should be treated with nucleus form factors and nuclear GPDs. With a magnitude of $10 \%$ for the interference term, the azimuthal modulation should be observable in the 3 cases.

Even in the last case of $\mathrm{Pb}$ beam on the $\mathrm{H}$ target, where we only expect 6000 events, the interference effect with its azimuthal modulation should be visible on top of the pure $\mathrm{BH}$ process. Assuming the same characteristics for muon detection as for the LHCb detector [37], one can proceed along the lines of [35] to evaluate the efficiency and the acceptance which could further reduce the 6000 events. In terms of single muon efficiency, one can take $97 \%$ as for the lowest $p_{T}$ muons studied in [36]. As regards the acceptance, one can adopt the same muon $p_{T}$ cut as in the exclusive $J / \psi$ studies, that is $p_{T, \mu}>400 \mathrm{MeV}$. It follows that, for the integration region $\Omega$ considered above, the acceptance is on the order of $80 \% .{ }^{15}$ Overall, one can thus reasonnably expect 4500 out of the 6000 events to be detected.

On the figure 8 , we show the magnitude of the 3 terms as function of the rapidity as well as the relative magnitude of the interference term with respect to the $\mathrm{BH}$ one for three cases of collisions (a) proton beam on a $\mathrm{Pb}$ target, (b) $\mathrm{Pb}$ beam on a $\mathrm{H}$ target, (c) proton beam on a $\mathrm{H}$ target. On the left panels, we present the rapidity dependence of the $\mathrm{BH}$, TCS and BH-TCS terms of differential cross sections $\frac{d \sigma}{d y d Q^{2} d t d \phi}$ evaluated at $Q^{2}=4 \mathrm{GeV}^{2}$, $t=-0.35 \mathrm{GeV}^{2}$ and $\phi=0$ and integrated over $\theta \in(\pi / 4,3 \pi / 4)$ for the GK model. On the right panels, we show the ratio of the interference signal to the BH for the GK and G-MSTW model. We see that for all cases the signal to the background ratio is around 10-15\%.

The simplest way to extract the size of the $\cos \phi$ azimuthal modulation, $B$, "defined" such that $d \sigma / d \phi=A+B \cos \phi$, would be to divide the sample in two bins in $\phi([-\pi / 2, \pi / 2]$ and $[\pi / 2,3 \pi / 2])$ and to measure the relative difference of the yield in these bins. The statistical relative uncertainty on $B$ is then trivially $\delta B / B=\pi / 2 \times A / B \times 1 / \sqrt{N_{\text {events }}}$.

\footnotetext{
${ }^{15}$ This number is higher than the acceptance of $J / \psi$ in the same conditions because the fiducial region $\Omega$ contains a cut on $\theta$ which selects out muons at rather large angles which therefore pass the $p_{T}$ cut more easily.
} 

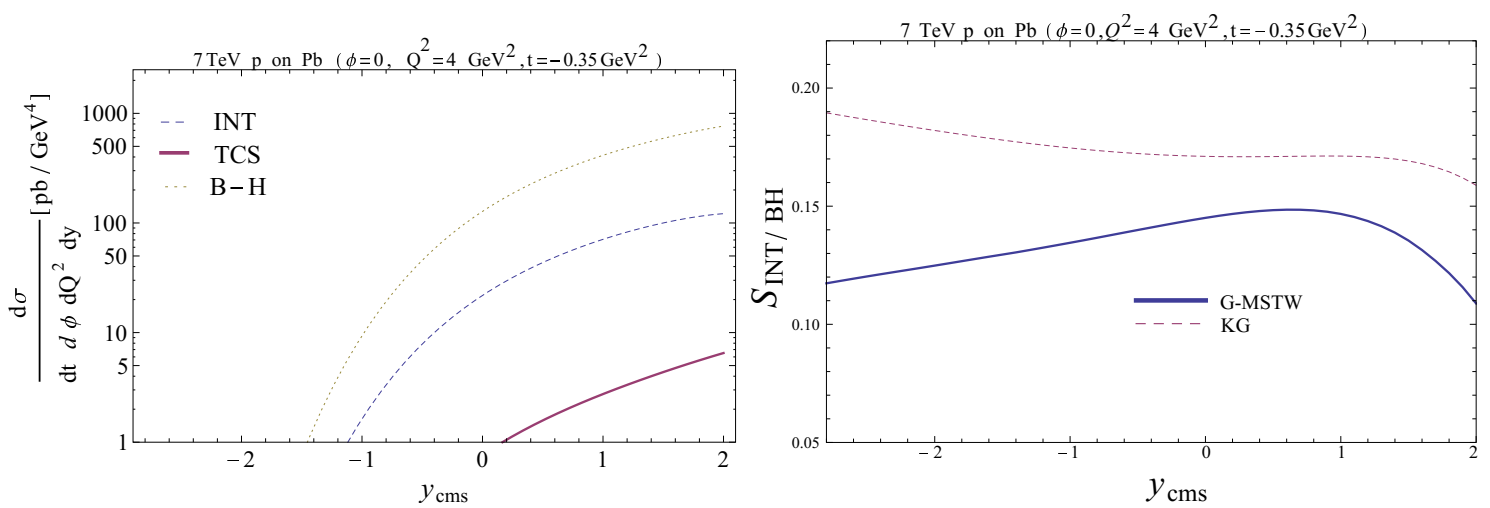

(a) $7 \mathrm{TeV} p$ on $\mathrm{Pb}$ target.
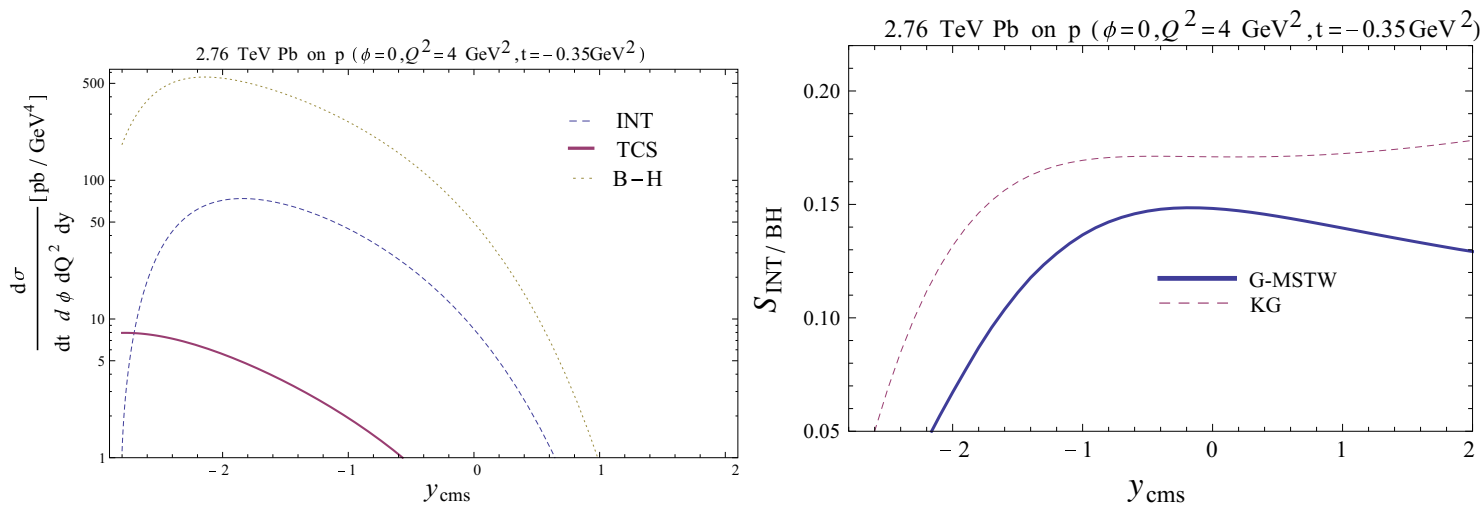

(b) $2.76 \mathrm{TeV} \mathrm{Pb}$ on $\mathrm{H}$ target.
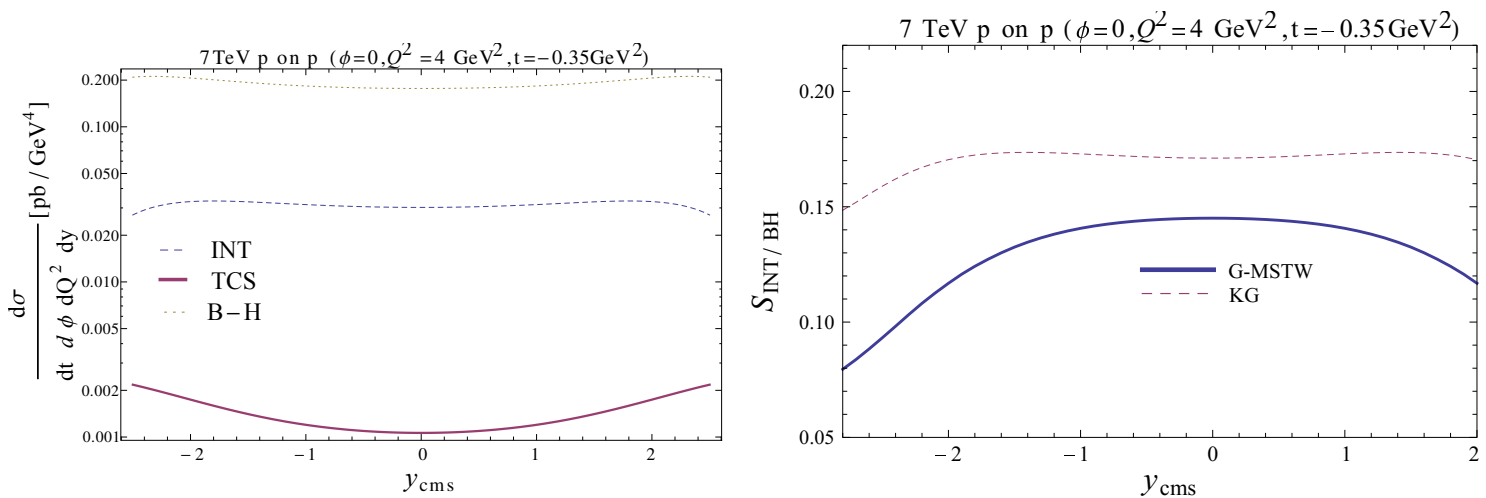

(c) $7 \mathrm{TeV} p$ on $\mathrm{H}$ target.

Figure 8. (Left) Differential cross section $\frac{d \sigma}{d y d t d \phi d Q^{2}}$ for the KG model for $Q^{2}=4 \mathrm{GeV}^{2}, t=$ $-0.35 \mathrm{GeV}^{2}$ and $\phi=0$ integrated over $\theta \in(\pi / 4,3 \pi / 4)$. Dotted line: B-H, dashed line: interference term, solid line: TCS. (Right) Ratio of the interference to BH differential cross section $\frac{d \sigma}{d y d t d \phi d Q^{2}}$ calculated for $Q^{2}=4 \mathrm{GeV}^{2}, t=-0.35 \mathrm{GeV}^{2}$ and $\phi=0$ for the GK (dashed) and G-MSTW (solid) models at NLO.From (a) to (c) $p \mathrm{~Pb}, \mathrm{PbH}$ and $p \mathrm{H}$ cases. 
With $B / A$ on the order of $10 \%$ as illustrated on the figure 8 and 4500 recorded events in the less favourable case, the coefficient of the modulation (i.e. the effect of the interference which is connected to the GPDs) can be measured with a relative precision of $25 \%$.

\section{Exclusive lepton-pair hadroproduction via odderon-pomeron fusion}

Throughout this work, we have based our discussion on the idea that the (theoretical and experimental) requirements for selecting UPC was de facto preventing hadronic interactions to take place or at least that photon-induced processes would be dominant. This is a rather safe assumption in nucleus-nucleus collisions where the coherent photon fluxes are enhanced by a factor $Z^{2}$ for each nucleus, whereas double-pomeron-induced reactions only scale like $A^{1 / 3}$. It also seems to be a reasonable assumption for $p A$ collisions that electromagnetic interactions dominate.

In the case of $p p$ collisions, the $Z^{2}$ factor is absent and the UPC requirements are also very similar, if not identical, in practice, to that for an exclusive or diffractive process. For instance, in order to impose $b>1 / R_{p}$, one can impose that the proton expected to emit the photon is only deflected by a $\Delta p_{T}$ of say maximum $100 \mathrm{MeV}$, which corresponds to $b \gtrsim 2 \mathrm{fm}$. Such a requirement however may not be sufficient to prevent any scattering by the exchange of a pomeron or an odderon, in more general terms a colourless QCD scattering as opposed to a QED scattering via one (or two) photon exchange.

The production of a lepton pair in $p p$ UPC via at least one photon exchange, which we discussed so far, should thus be confronted to a potentially competing process leading to the same final state, in which the virtual photon decaying into lepton pair is produced in an Odderon-Pomeron $(\mathbb{O P})$ fusion as depicted in figure $3 \mathrm{~d}$. In the case of the photon-pomeron $(\gamma \mathbb{P})$ process depicted on figure 3c, such a scattering should in principle be encompassed in the GPD description at high energies and it is not expected to qualitatively change our predictions. It is rather a matter of a different modelling of the GPD.

In contrast, the production from an $\mathbb{O P}$ fusion cannot be recast into any kind of photoninduced contribution. In the case of exclusive vector-meson production in $p p$ collisions (see below), the $\mathbb{O P}$ fusion can directly compete with the $\gamma \mathbb{P}$ contribution. Strangely enough, to the best of our knowledge, it has never considered before in the context of the exclusive production of a dilepton pair. A reason why nobody looked at it previously is its likely suppression with respect to the dominant $\mathrm{BH}$ contribution. However, in the context of GPD studies, one in any case needs to study sub-dominant contributions giving rise to azimuthal modulations, as the TCS QCD contribution. It thus makes perfect sense to compare the TCS and $\mathbb{O P}$ fusion contributions.

Yet, as we will briefly discuss below, the evaluation of the latter is a non-trivial task equivalent to another one-loop computations - and which necessarily involves unknown nonperturbative unintegrated parton distributions, the so-called proton impact factors, which makes the predictions essentially model-dependent and probably precludes one to draw any definite quantitative conclusions. 


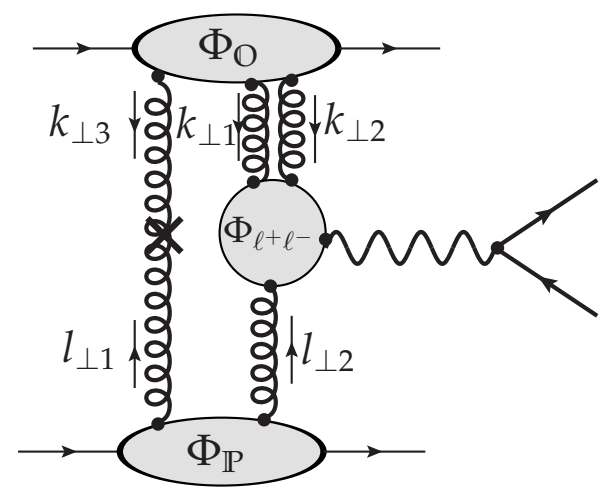

Figure 9. The lowest order diagram contributing to the non-factorised $\mathbb{O P}$ fusion for lepton-pair production. The gluons with the momenta $l_{\perp 1}, l_{\perp 2}$ carry the colour indices $\lambda_{1}, \lambda_{2}$, respectively and those with the momenta $k_{\perp 1}, k_{\perp 2}, k_{\perp 3}$ carry the colour indices $\kappa_{1}, \kappa_{2}, \kappa_{3}$.

Indeed, the natural — and most tractable — framework to study $\mathbb{O P}$ fusion at high energies is the $k_{T}$-factorisation (see e.g. [40]) where the scattering amplitude, with the momenta defined as depicted on figure 9 , reads:

$$
\begin{gathered}
\mathcal{M}_{\mathbb{O P}}=-i s \frac{2 \cdot 3}{2 ! 3 !} \frac{4}{(2 \pi)^{8}} \int \frac{d^{2} l_{\perp 1}}{l_{\perp_{1}}^{2}} \frac{d^{2} l_{\perp 2}}{l_{\perp 2}^{2}} \delta^{2}\left(l_{\perp_{1}}+l_{\perp_{2}}-l_{\perp}\right) \frac{d^{2} k_{\perp 1}}{k_{\perp 1}^{2}} \frac{d^{2} k_{\perp 2}}{k_{\perp 2}^{2}} \frac{d^{2} k_{\perp 3}}{k_{\perp 3}^{2}} \delta^{2}\left(k_{\perp_{1}}+k_{\perp_{2}}+k_{\perp_{3}}-k_{\perp}\right) \\
\times \delta^{2}\left(k_{\perp 3}+l_{\perp 1}\right) k_{\perp 3}^{2} \delta^{\lambda_{1} \kappa_{3}} \cdot \Phi_{\mathbb{P}}^{\lambda_{1} \lambda_{2}}\left(l_{\perp_{1}}, l_{\perp_{2}}\right) \cdot \Phi_{\mathbb{O}}^{\kappa_{1} \kappa_{2} \kappa_{3}}\left(k_{\perp_{1}}, k_{\perp_{2}}, k_{\perp_{3}}\right) \cdot \Phi_{\ell^{+} \ell^{-}}^{\lambda_{2} \kappa_{2}}\left(l_{\perp_{2}}, k_{\perp_{1}}, k_{\perp_{2}}\right),
\end{gathered}
$$

where $\Phi_{\mathbb{P}}^{\lambda_{1} \lambda_{2}}\left(l_{\perp 1}, l_{\perp 2}\right)$ denotes the impact factor of the proton, scattered via an (unfactorised) pomeron exchange and $\Phi_{\mathbb{O}}^{\kappa_{1} \kappa_{2} \kappa_{3}}\left(k_{\perp 1}, k_{\perp 2}, k_{\perp 3}\right)$ denotes the impact factor of the proton, scattered via an (unfactorised) odderon exchange. $\Phi_{\ell^{+} \ell^{-}}^{\lambda_{2} \kappa_{2}}\left(l_{\perp 2}, k_{\perp 1}, k_{\perp 2}\right)$ is the effective production vertex of the $\ell^{+} \ell^{-}$pair. It results from the fusion of a gluon with the transverse momentum and the colour index $\left(l_{\perp 2}, \lambda_{2}\right)$ from the pomeron with two gluons $\left(k_{\perp 1}, \kappa_{1}\right)$ and $\left(k_{\perp 2}, \kappa_{2}\right)$ of the odderon. The factor $\frac{2 \cdot 3}{2 ! 3 !}=\frac{1}{2}$ is a combinatorial factor and $\frac{1}{2 !}$ as well as $\frac{1}{3 !}$ correct the over-counting of diagrams introduced by the factorisation in the scattering amplitudes of the impact factor from the pomeron and odderon exchanges, respectively. The factor $2 \cdot 3=6$ accounts for all the possibilities to build the spectator gluon from the momenta $l_{\perp_{i}}$ and $k_{\perp j}$. Finally, the factor $\delta^{2}\left(k_{\perp_{3}}+l_{\perp_{1}}\right) k_{\perp_{3}}^{2} \delta^{\lambda_{1} \kappa_{3}}$ comes from an artificial vertex (denoted by the cross in figure 9) introduced to keep the notations of the momenta $l_{\perp i}$ and $k_{\perp j}$ as symmetric as possible and which connects the spectator gluons $\left(l_{\perp 1}, \lambda_{1}\right)$ and $\left(k_{\perp 3}, \kappa_{3}\right)$.

The effective vertex $\Phi_{\ell^{+} \ell^{-}}^{\lambda_{2} \kappa_{1} \kappa_{2}}\left(l_{\perp 2}, k_{\perp 1}, k_{\perp 2}\right)$ can be computed as the DIS impact factor, $\gamma^{\star} \rightarrow \gamma^{\star}$, or the hard scattering for $\gamma^{\star} g \rightarrow \gamma g$ within the $k_{T}$-factorisation approach $[38,39]$. It effectively amounts to a non-trivial one-loop computation. Indeed, it can be rewritten as the integration, over the Sudakov variable ${ }^{16} \beta_{k_{1}}$, of the $S$-matrix element of the transition $g\left(k_{\perp 1}\right) g\left(k_{\perp 2}\right) g\left(l_{\perp 2}\right) \rightarrow \ell^{+} \ell^{-}$with off-shell gluons, ${ }^{17}$

$$
\Phi_{\ell^{+} \ell^{-}}^{\lambda_{2} \kappa_{1} \kappa_{2}}\left(l_{\perp 2}, k_{\perp 1}, k_{\perp 2}\right)=\int d \beta_{k_{1}} \mathcal{S}_{\mu_{2}^{\prime} \nu_{1}^{\prime} \nu_{2}^{\prime}}^{\lambda_{2} \kappa_{2} \kappa_{2}}\left(g\left(k_{\perp 1}\right) g\left(k_{\perp 2}\right) g\left(l_{\perp 2}\right) \rightarrow \ell^{+} \ell^{-}\right) \frac{p_{2}^{\mu_{2}^{\prime}} p_{1}^{\nu_{1}^{\prime}} p_{1}^{\nu_{2}^{\prime}}}{s} .
$$

${ }^{16} k_{i}^{\mu} \simeq \beta_{k_{i}} p_{1}^{\mu}+k_{\perp i}^{\mu}$ and $l_{i}^{\mu} \simeq \beta_{l_{i}} p_{2}^{\mu}+l_{\perp i}^{\mu}$ with $i=1,2,3$.

${ }^{17}$ With their longitudinal polarisation vectors expressed in terms of the incoming proton momenta. 

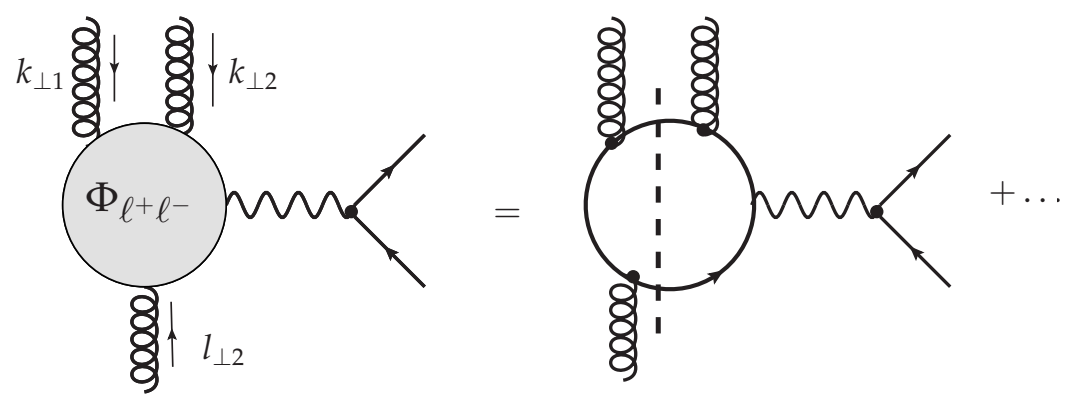

Figure 10. The hard vertex for the lepton-pair production in the non-factorised $\mathbb{O P}$ fusion. The sum $+\ldots$ denotes the remaining 3 cut diagrams contributing at the lowest order in $\alpha_{s}$. The dotted line denotes the $s$-channel cut expected to give the dominant contribution in the small- $x$ regime.

This computation is not straightforward ${ }^{18}$ and beyond the scope of this discussion, but one can emphasise some generic properties of the amplitude which are of specific relevance. Although the impact factors $\Phi_{\mathbb{P}}$ and $\Phi_{\mathbb{O}}$ are essentially non-perturbative objects which have to be modelled, we know that they should be real functions of the external momenta. In the kinematical domain of small- $x$ (high energies), $\Phi_{\ell^{+} \ell^{-}}^{\lambda_{2} \kappa_{2}}\left(l_{\perp 2}, k_{\perp 1}, k_{\perp 2}\right)$ develops a dominant imaginary part determined by the Cutkosky cut (the dashed line on figure 10). One may therefore think that the vertex is essentially a real function of the gluon momenta as in the case of DIS or the electroproduction of charmonium [40]. Yet, due to the positive virtuality of the photon $\left(Q^{2}>0\right)$, the quark propagator between $\gamma^{\star}$ and the gluon with momentum $k_{\perp 2}$ on figure 10 can also develop an additional absorptive part. As a consequence, we have no reason to exclude that the present effective vertex is in fact a complex function of the gluon momenta. As such, it can also generate, through its interference with the BH contribution, azimuthal asymmetries. If the amplitude of the $\mathbb{O P}$ fusion contribution had been purely real, it would have had de facto no impact on the extraction of the GPD in the $p p$ case. Unfortunately, only a dedicated study, well beyond the scope of this article, could tell more on the impact of the $\mathbb{O P}$ fusion process.

Yet, further statements can be made, still on the qualitative level. In particular, the evaluation of the $\mathbb{O P}$ fusion contribution to the central exclusive hadroproduction of a $J / \psi$ studied in [40] is similar and also involves the nonperturbative impact factors $\Phi_{\mathbb{P}}$ and $\Phi_{\mathbb{O}}$. Given that the quantum numbers of a $J / \psi$ and a photon are the same, one can expect a similar qualitative behaviour of the scattering amplitudes in both cases. In the $J / \psi$ case, the production can also result from the $\gamma \mathbb{P}$ fusion - that is the one expected from photon-induced UPC which could also be expressed in terms of gluon GPDs. In fact, it was found in [40] that the cross sections for both processes are of similar magnitude at the Tevatron. ${ }^{19}$ Both the $\mathbb{O P}$ and $\gamma \mathbb{P}$ production mechanisms were however also found to be characterised by a different $J / \psi$ transverse-momentum $(t)$ dependence. In particular, if one imposes that the final state protons have $|t|>0.25 \mathrm{GeV}^{2}$, the $\mathbb{O P}$ contribution can be enhanced ten times with respect to the photon induced one (see table 1 of [40]).

\footnotetext{
${ }^{18}$ It involves for instance a double integral on Feynman variables.

${ }^{19}$ Note that owing to the increase of the gap survival probability for lower energies, it is reasonable to believe that, at AFTER@LHC energies, i.e. around $\sqrt{s_{N N}}=115 \mathrm{GeV}$, exclusive $J / \psi$ production in $p p$ collisions would be dominated by $\mathbb{O P}$ fusion.
} 
In the case of the lepton-pair production in the conditions of UPC, we expect a similar behaviour. Provided that the difference in the vertex function $\Phi_{\ell^{+} \ell^{-}}$does not alter the dominant momentum-transfer region in its convolution with the impact factors, the $t$ dependence of the $\mathbb{O P}$ fusion contribution should also follow from that of the impact factors, $\Phi_{\mathbb{P}}$ and $\Phi_{\mathbb{O}}$, which are common to both $J / \psi$ and $\gamma^{\star}$ production. Imposing a cut on $t$ may thus isolate the $\mathbb{O P}$ fusion contribution from the photon induced one and, in a sense, provide an unexpected path to the Odderon discovery via its interference with the pure QED $\mathrm{BH}$ process in the same way as the TCS signal would be extracted in the region of low $|t|$.

As recently discussed in [33], the odderon could also contribute to the $\eta_{c}$ production. Due to its different quantum numbers with respect to the $J / \psi$, the $\eta_{c}$ can be produced by photon-odderon $(\gamma \mathbb{O})$ fusion. In the $p p$ case, the cross section, which we obtained from $\gamma \gamma$ fusion, is slightly larger than that from $\gamma \mathbb{O}$ fusion as obtained in [33], both on the order of a picobarn or less. In the $p \mathrm{~Pb}$ and $\mathrm{Pb} p$ cases, the photon can be radiated by the ion and the odderon by the proton. As we noted, although $m_{\eta_{c}}$ is in principle above the so-called energy "cut-off", the photon flux is not zero, even when accounting from the minimum impact parameter for a proton-lead UPC. In the $\mathrm{Pb} p$ case, the $\gamma \mathbb{O}$ induced cross section ranges from 30 up to $360 \mathrm{pb}$ whereas the $\gamma \gamma$ induced one is on the order of $440 \mathrm{pb}$. As for the dilepton case, a study of the transverse-momentum dependence should be able to discriminate between both processes.

\section{Conclusion}

We have theoretically investigated the feasibility of accessing the lepton-pair production in ultraperipheral collisions at the proposed fixed-target experiment AFTER@LHC, which takes advantages of the multi-TeV proton and ion beams of the LHC. To this aim, we have first estimated the magnitude of the cross section for the lepton-pair production from the fusion of two quasi-real photons emitted by the quasi-grazing hadrons. This purely electromagnetic $\mathrm{BH}$ process can serve as an important tool for the determination of the luminosities with nucleon or ion beams but it can also be used for an experimental verification of the validity of the effective-photon approximation usually applied to estimate the flux of quasi-real photons emitted by these relativistically moving charges. The lepton-pair production also gives access to the proton GPDs via the TCS process. Another way to probe the photon flux is to measure $\eta_{c}$ production for which the production rate in $p p$ collisions at AFTER@LHC does depend on the minimal impact parameter used for the UPC.

The predictions which we obtained for the cross section for $\mathrm{BH}$ - using specific cuts relevant for the GPD extraction - are on the order of few thousand of $\mathrm{pb}$ for the $p \mathrm{~Pb}$ and $\mathrm{Pb} p$ cases and a slightly less than $10 \mathrm{pb}$ for the $p p$ case and we confirm the dominance of $\mathrm{BH}$ over TCS. This dominance can partially be overcome by studying of the interference - also sensitive on the GPDs - between TCS and BH which we evaluated at NLO. With specific cuts on the lepton polar angle, the ratio of this interference over the $\mathrm{BH}$ amplitude squared is on order of $10 \%$ with two models of GPDs, i.e. GK and G-MSTW. These are quite promising values giving hope for the extraction of this interference by means of the analysis of the azimuthal distribution of the produced leptons with, in the less favourable 
case, a relative precision of $25 \%$. Studying TCS in ultraperipheral collisions at a fixedtarget experiment can also give us opportunity to study target polarization asymmetries, which are an useful tool to extract further information on GPDs [41].

We have also derived cross sections for $\eta_{c}$ production by photon-pair fusion, which happens, in particular in this energy range, to be sensitive on the method used to compute the photon flux in the $p p$ case. We found out that $10^{4} \eta_{c}$ can be produced per year in UPCs with AFTER@LHC.

Finally, we discussed possible competing hadronic processes via pomeron or odderon exchanges which could interestingly be separated out by a careful analysis of the transversemomentum dependence of the produced particles. To our knowledge, this is the first time that such a pomeron-odderon-fusion contribution to exclusive dilepton production has been discussed, even qualitatively.

In conclusion, AFTER@LHC offers a realistic possibility to study lepton-pair production in ultraperipheral collisions which opens the path to investigate features of the partonic structure of hadrons which are complementary to those studied with lepton beams. The use of hadron beams may, for instance, offer the opportunity study to odderon-sensitive reactions.

\section{Acknowledgments}

We thank D. d'Enterria, V.P. Gonçalves, S. Klein, R. Mikkelsen, J. Nystrand for useful discussions. We are indebted to B. Trzeciak for the evaluation of the acceptance quoted in section 4. This work is partly supported by the COPIN-IN2P3 Agreement, the Polish Grant NCN No DEC-2011/01/D/ST2/02069, and the CNRS grants PICS-06149 Torino-IPNO \& PEPS4AFTER2.

\section{A Kinematics}

We denote the momenta of incoming nucleons (in the nucleon-nucleon $\mathrm{cms}$ ) as:

$$
\begin{aligned}
& p_{A}=\frac{\sqrt{s}}{2}(1,0,0, \alpha), \\
& p_{B}=\frac{\sqrt{s}}{2}(1,0,0,-\alpha),
\end{aligned}
$$

where the $A$ is a nucleon from the beam, $B$ is a nucleon from target and $\alpha=\sqrt{1-\frac{4 M^{2}}{s}}$. The Weizsäcker - Williams photon is emitted from a beam $(\epsilon=-1)$ or a target nucleon $(\epsilon=+1)$, and its momentum is given by:

$$
k=x_{\gamma} \frac{\sqrt{s}}{2}(1,0,0,-\epsilon)
$$

Momentum of outgoing lepton pair (or outgoing virtual photon decaying into heavy lepton pair) reads:

$$
q=\left(q_{0}, q_{T}, q_{z}\right) \equiv\left(m_{T} \cosh y^{\ell \ell}, q_{T}, m_{T} \sinh y^{\ell \ell}\right),
$$


where $m_{T}=\sqrt{q_{T}^{2}+Q^{2}}$ and $y^{\ell \ell}$ is the lepton-pair rapidity which can be expressed as

$$
y^{\ell \ell}=\frac{1}{2} \epsilon \log \left(\frac{\left(Q^{2}-t\right)(\alpha+1)}{Q^{2}(\alpha-1)-t\left(\alpha-1-2 x_{\gamma}\right)+s x_{\gamma}^{2}(\alpha+1)}\right),
$$

where:

$$
t \equiv(k-q)^{2}=Q^{2}-m_{T} \sqrt{s} x_{\gamma} e^{\epsilon y^{\ell \ell}} .
$$

Inverting eq. (A.4), we can express $x_{\gamma}$ as a function of $y^{\ell \ell}$ :

$$
x_{\gamma}\left(y^{\ell \ell}, t, Q, \epsilon\right)=\frac{-2 t+\sqrt{4 t^{2}-4 s\left(Q^{2}-t\right)(\alpha+1)\left[(\alpha-1)-(\alpha+1) e^{-2 \epsilon y^{\ell \ell}}\right]}}{2 s(\alpha+1)} .
$$

or combining eq. (A.5) with eq. (A.6) we easily get:

$$
\begin{aligned}
t & =t\left(y^{\ell \ell}, q_{T}^{2}, Q, \epsilon\right), \\
x_{\gamma} & =x_{\gamma}\left(y^{\ell \ell}, q_{T}^{2}, Q, \epsilon\right), \\
J & =\frac{d t}{d q_{T}^{2}} .
\end{aligned}
$$

\section{B Compton form factors and generalised parton distributions}

In this appendix, we give the expressions of the LO hard-scattering kernel $T_{i}$ appearing in the expression of the Compton form factor $\mathcal{H}, \tilde{\mathcal{H}}$ and $\tilde{\mathcal{E}}$. At Born order, the hard-scattering kernel associated to the quark GPDs are given by

$$
\begin{aligned}
& T_{H}^{q}(x, \eta)=e_{q}^{2}\left(\frac{1}{-\eta-x-i \epsilon}-\frac{1}{-\eta+x-i \epsilon}\right), \\
& T_{\tilde{H}}^{q}(x, \eta)=e_{q}^{2}\left(\frac{1}{-\eta-x-i \epsilon}+\frac{1}{-\eta+x-i \epsilon}\right), \\
& T_{E}^{q}(x, \eta)=e_{q}^{2}\left(\frac{1}{-\eta-x-i \epsilon}-\frac{1}{-\eta+x-i \epsilon}\right),
\end{aligned}
$$

and those associated to the gluon GPDs are zero. The NLO hard-scattering kernels can be found in $[10,34]$.

Open Access. This article is distributed under the terms of the Creative Commons Attribution License (CC-BY 4.0), which permits any use, distribution and reproduction in any medium, provided the original author(s) and source are credited.

\section{References}

[1] STAR collaboration, C. Adler et al., Coherent $\rho^{0}$ production in ultraperipheral heavy ion collisions, Phys. Rev. Lett. 89 (2002) 272302 [nucl-ex/0206004] [INSPIRE].

[2] STAR collaboration, J. Adams et al., Production of $e^{+} e^{-}$pairs accompanied by nuclear dissociation in ultra-peripheral heavy ion collision, Phys. Rev. C 70 (2004) 031902 [nucl-ex/0404012] [INSPIRE]. 
[3] PHENIX collaboration, S. Afanasiev et al., Photoproduction of $J / \psi$ and of high mass $e^{+} e^{-}$ in ultra-peripheral Au+Au collisions at $\sqrt{s}=200$ GeV, Phys. Lett. B 679 (2009) 321 [arXiv:0903.2041] [INSPIRE].

[4] ALICE collaboration, Coherent $\mathrm{J} / \psi$ photoproduction in ultra-peripheral $\mathrm{Pb}$ - $\mathrm{Pb}$ collisions at $\sqrt{s_{N N}}=2.76$ TeV, Phys. Lett. B 718 (2013) 1273 [arXiv:1209.3715] [INSPIRE].

[5] ALICE collaboration, Charmonium and $e^{+} e^{-}$pair photoproduction at mid-rapidity in ultra-peripheral Pb-Pb collisions at $\sqrt{s_{\mathrm{NN}}}=2.76 \mathrm{TeV}$, Eur. Phys. J. C 73 (2013) 2617 [arXiv: 1305.1467] [INSPIRE].

[6] S.J. Brodsky, F. Fleuret, C. Hadjidakis and J.P. Lansberg, Physics opportunities of a fixed-target experiment using the LHC beams, Phys. Rept. 522 (2013) 239 [arXiv:1202.6585] [INSPIRE].

[7] H. Bethe and W. Heitler, On the stopping of fast particles and on the creation of positive electrons, Proc. Roy. Soc. Lond. A 146 (1934) 83 [InSPIRE].

[8] E.R. Berger, M. Diehl and B. Pire, Time-like Compton scattering: exclusive photoproduction of lepton pairs, Eur. Phys. J. C 23 (2002) 675 [hep-ph/0110062] [INSPIRE].

[9] B. Pire, L. Szymanowski and J. Wagner, Can one measure timelike Compton scattering at LHC?, Phys. Rev. D 79 (2009) 014010 [arXiv:0811.0321] [InSPIRE].

[10] B. Pire, L. Szymanowski and J. Wagner, NLO corrections to timelike, spacelike and double deeply virtual Compton scattering, Phys. Rev. D 83 (2011) 034009 [arXiv:1101.0555] [INSPIRE].

[11] H. Moutarde, B. Pire, F. Sabatie, L. Szymanowski and J. Wagner, Timelike and spacelike deeply virtual Compton scattering at next-to-leading order, Phys. Rev. D 87 (2013) 054029 [arXiv: 1301.3819] [INSPIRE].

[12] W. Schafer, G. Slipek and A. Szczurek, Exclusive diffractive photoproduction of dileptons by timelike Compton scattering, Phys. Lett. B 688 (2010) 185 [arXiv:1003.0610] [INSPIRE].

[13] D. Müller, D. Robaschik, B. Geyer, F.M. Dittes and J. Hořejši, Wave functions, evolution equations and evolution kernels from light ray operators of QCD, Fortsch. Phys. 42 (1994) 101 [hep-ph/9812448] [INSPIRE].

[14] X.-D. Ji, Gauge-invariant decomposition of nucleon spin, Phys. Rev. Lett. 78 (1997) 610 [hep-ph/9603249] [INSPIRE].

[15] A.V. Radyushkin, Nonforward parton distributions, Phys. Rev. D 56 (1997) 5524 [hep-ph/9704207] [INSPIRE].

[16] J.C. Collins and A. Freund, Proof of factorization for deeply virtual Compton scattering in QCD, Phys. Rev. D 59 (1999) 074009 [hep-ph/9801262] [INSPIRE].

[17] M. Diehl, Generalized parton distributions, Phys. Rept. 388 (2003) 41 [hep-ph/0307382] [INSPIRE].

[18] A.V. Belitsky and A.V. Radyushkin, Unraveling hadron structure with generalized parton distributions, Phys. Rept. 418 (2005) 1 [hep-ph/0504030] [INSPIRE].

[19] S. Boffi and B. Pasquini, Generalized parton distributions and the structure of the nucleon, Riv. Nuovo Cim. 30 (2007) 387 [arXiv:0711.2625] [INSPIRE]. 
[20] M. Guidal, H. Moutarde and M. Vanderhaeghen, Generalized parton distributions in the valence region from deeply virtual Compton scattering, Rept. Prog. Phys. 76 (2013) 066202 [arXiv:1303.6600] [INSPIRE].

[21] V.M. Budnev, I.F. Ginzburg, G.V. Meledin and V.G. Serbo, The two photon particle production mechanism. Physical problems. Applications. Equivalent photon approximation, Phys. Rept. 15 (1975) 181 [INSPIRE].

[22] A.J. Baltz, The physics of ultraperipheral collisions at the LHC, Phys. Rept. 458 (2008) 1 [arXiv:0706.3356] [INSPIRE].

[23] J.P. Lansberg et al., A fixed-target experiment at the LHC (AFTER@LHC): luminosities, target polarisation and a selection of physics studies, PoS (QNP2012) 049 [arXiv:1207.3507] [INSPIRE].

[24] A. Rakotozafindrabe et al., Spin physics at a fixed-target experiment at the LHC (AFTER@LHC), Phys. Part. Nucl. 45 (2014) 336 [arXiv:1301.5739] [InSPIRE].

[25] L. Massacrier et al., Studies of transverse-momentum-dependent distributions with a fixed-target experiment using the LHC beams (AFTER@LHC), arXiv:1502.00984 [INSPIRE].

[26] R.E. Mikkelsen, A.H. Sørensen and U.I. Uggerhøj, Bremsstrahlung from relativistic heavy ions in a fixed target experiment at the LHC, arXiv:1503.06621 [INSPIRE].

[27] M. Drees and D. Zeppenfeld, Production of supersymmetric particles in elastic ep collisions, Phys. Rev. D 39 (1989) 2536 [inSPIRE].

[28] M. Drees, R.M. Godbole, M. Nowakowski and S.D. Rindani, $\gamma \gamma$ processes at high-energy pp colliders, Phys. Rev. D 50 (1994) 2335 [hep-ph/9403368] [INSPIRE].

[29] G. Baur, K. Hencken, D. Trautmann, S. Sadovsky and Y. Kharlov, Coherent $\gamma \gamma$ and $\gamma A$ interactions in very peripheral collisions at relativistic ion colliders, Phys. Rept. 364 (2002) 359 [hep-ph/0112211] [INSPIRE].

[30] G. Racah, On pair production through charged particle collisions, Nuovo Cim. 14 (1937) 93 [INSPIRE].

[31] A.J. Baltz, Y. Gorbunov, S.R. Klein and J. Nystrand, Two-photon interactions with nuclear breakup in relativistic heavy ion collisions, Phys. Rev. C 80 (2009) 044902 [arXiv: 0907.1214] [INSPIRE].

[32] Particle Data Group collaboration, K.A. Olive et al., Review of particle physics, Chin. Phys. C 38 (2014) 090001 [InSPIRE].

[33] V.P. Goncalves and W.K. Sauter, $\eta_{c}$ production in photon-induced interactions at a fixed target experiment at LHC as a probe of the odderon, Phys. Rev. D 91 (2015) 094014 [arXiv: 1503.05112] [INSPIRE].

[34] D. Mueller, B. Pire, L. Szymanowski and J. Wagner, On timelike and spacelike hard exclusive reactions, Phys. Rev. D 86 (2012) 031502 [arXiv:1203.4392] [inSPIRE].

[35] L. Massacrier et al., Feasibility studies for quarkonium production at a fixed-target experiment using the LHC proton and lead beams (AFTER@LHC), arXiv:1504.05145 [INSPIRE].

[36] F. Archilli et al., Performance of the muon identification at LHCb, 2013 JINST 8 P10020 [arXiv: 1306.0249] [INSPIRE]. 
[37] LHCb collaboration, Updated measurements of exclusive $J / \psi$ and $\psi(2 S)$ production cross-sections in pp collisions at $\sqrt{s}=7 \mathrm{TeV}$, J. Phys. G 41 (2014) 055002 [arXiv: 1401.3288] [INSPIRE].

[38] V.N. Gribov, L.N. Lipatov and G.V. Frolov, The leading singularity in the $j$ plane in quantum electrodynamics, Sov. J. Nucl. Phys. 12 (1971) 543 [Yad. Fiz. 12 (1970) 994] [INSPIRE].

[39] I. Balitsky and E. Kuchina, Deeply virtual Compton scattering at small x, Phys. Rev. D 62 (2000) 074004 [hep-ph/0002195] [INSPIRE].

[40] A. Bzdak, L. Motyka, L. Szymanowski and J.R. Cudell, Exclusive $J / \psi$ and $\Upsilon$ hadroproduction and the QCD odderon, Phys. Rev. D 75 (2007) 094023 [hep-ph/0702134] [INSPIRE].

[41] M. Boër, M. Guidal and M. Vanderhaeghen, Single and double polarization observables in timelike Compton scattering off proton, arXiv:1501.00270 [INSPIRE]. 